\title{
Sexual Selection and Population Divergence I. the InflUenCE OF SOCIALly FleXIBLE CUTICULAR Hydrocarbon Expression In MALE FIELd CRICKETS (TELEOGRYLLUS OCEANICUS)
}

Sonia Pascoal ${ }^{1}$, Magdalena Mendrok ${ }^{2}$, Christopher Mitchell ${ }^{3,4}$, Alastair J. Wilson ${ }^{4}$, John Hunt $^{4}$, Nathan W. Bailey ${ }^{1,5}$

${ }^{1}$ Centre for Biological Diversity, University of St Andrews, St Andrews, KY16 9TH, United Kingdom

${ }^{2}$ Institute of Environmental Sciences, Jagellonian University, Gronostajova 7, 30-387 Kraków, Poland

${ }^{3}$ Hawkesbury Institute for the Environment, University of Western Sydney, Penrith NSW 2751, Australia

${ }^{4}$ Centre for Ecology and Conservation, College of Life and Environmental Sciences, University of Exeter, Cornwall Campus, TR10 9EZ, United Kingdom

${ }^{5}$ E-mail:nwb3@st-andrews.ac.uk

Running Title: Divergence and Social Flexibility

Data Archive Location: Microsatellite allele frequencies, cuticular hydrocarbon profiles, calling song data, and morphological measurements are archived at Dryad Digital Repository, doi:10.5061/dryad.tb552. 


\section{Abstract}

Debates about how coevolution of sexual traits and preferences might promote evolutionary diversification have permeated speciation research for over a century. Recent work demonstrates that the expression of such traits can be sensitive to variation in the social environment. Here we examined social flexibility in a sexually selected male trait cuticular hydrocarbon $(\mathrm{CHC})$ profiles - in the field cricket Teleogryllus oceanicus and tested whether population genetic divergence predicts the extent or direction of social flexibility in allopatric populations. We manipulated male crickets' social environments during rearing and then characterised $\mathrm{CHC}$ profiles. $\mathrm{CHC}$ signatures varied considerably across populations and also in response to the social environment, but our prediction that increased social flexibility would be selected in more recently founded populations exposed to fluctuating demographic environments was unsupported. Furthermore, models examining the influence of drift and selection failed to support a role of sexual selection in driving population divergence in $\mathrm{CHC}$ profiles. Variation in social environments might alter the dynamics of sexual selection, but our results align with theoretical predictions that the role social flexibility plays in modulating evolutionary divergence depends critically on whether responses to variation in the social environment are homogeneous across populations, or whether gene-by-social-environment interactions occur.

KEY WORDS: Founder effect, gene-by-social environment interaction, interacting phenotype, social flexibility, range expansion 


\section{Introduction}

Identifying evolutionary forces that cause phenotypic and genetic divergence among isolated populations is a fundamental goal of evolutionary biology. Sexual signalling traits are obvious candidates as substrates for selection to act upon in this context: populationlevel variation in the dynamics of genetic drift or the action of selection on traits involved in sexual reproduction can cause reproductive isolation, which feeds back to strengthen divergence (Ritchie 2007). However, the expression of secondary sexual traits appears to be particularly susceptible to variation in the social environment (Rodríguez et al. 2013b).

Social flexibility can be caused by processes such as imprinting of sexual preferences during juvenile stages, learning about the abundance and quality of conspecifics in the immediate environment, or more passive effects such as habituation, copying, or density-dependence. Here we test the relationship between social flexibility and the evolutionary divergence of sexual traits across populations.

The role of behavioural flexibility in population divergence is debated (e.g. Baldwin 1896; West-Eberhard 1989; Miller and Svensson 2014). Recent work mostly focuses on how learning influences reproductive isolation, for example by relaxing, strengthening, or changing the direction of mating preferences (Servedio et al. 2009; Svensson et al. 2010; Verzijden et al. 2012). Traits that are responsive to the social environment but do not involve learning might also cause unusual evolutionary dynamics (Wolf et al. 2014). For example, when genetic variation among individuals causes variation in the social environment, indirect genetic effects (IGEs) can cause feedback that alters evolutionary rates and directions of interacting phenotypes (Moore et al. 1997). A substantial theoretical 
literature has developed to model the impact of IGEs on evolutionary processes such as sexual conflict and sexual selection (Moore and Pizzari 2005; Bailey and Moore 2012; Bijma 2014). This literature suggests that social flexibility may play an important role in shaping patterns of genetic divergence in spatially separated populations, possibly by causing variation in selection on traits that contribute to reproductive isolation (Agrawal et al. 2001; Bailey and Moore 2012). Recent empirical work confirms the existence of IGEs on sexuallyselected traits such as male cuticular hydrocarbons in Drosophila serrata (Petfield et al. 2005) and female choice in the field cricket Teleogryllus oceanicus (Bailey and Zuk 2012). Of particular interest are genotype-by-social environment interactions (GSEIs), in which different focal genotypes respond differently to variation in the social environment (Wolf et al. 2014). If GSEIs operate on sexual signal expression among allopatric populations of a species, for example, alternative rates and directions of evolutionary change could accelerate the evolution of reproductive isolation under some conditions.

Less is known about whether social flexibility enables individuals to cope with demographic changes upon colonising a new habitat. The ability to facultatively adjust phenotypes to best suit the prevailing social conditions can be adaptive in the context of demographic stochasticity. For instance, social flexibility can confer an advantage to an individual if the distribution of mating partners is unpredictable and greater reproductive fitness can be achieved by optimising courtship behaviour and/or mate choice depending on their immediate availability (Dukas 2008; Kasumovic and Brooks 2011). Such demographic stochasticity is likely after dispersal or migration and can have a large impact on subsequent population growth and evolution (Szücs et al. 2014). Those individuals that can respond adaptively to different social environments may be favoured in more recently founded 
populations. This could occur either because the most socially responsive genotypes present in the source population are more likely to survive and reproduce in a founding population, or because longer-term demographic instability in colonising populations might favour new mutations that confer greater social flexibility (Whitlock 1992). Either mechanism leads to a directional prediction of greater social flexibility in more recently established populations, which can be tested by comparing social flexibility in populations with different colonisation histories. In contrast, source populations with comparatively stable demographics are expected to experience relaxed selection with respect to socially-flexible phenotypes because plasticity is commonly thought to impose fitness costs (DeWitt et al. 1998). Our prediction is that on average, individuals from more demographically stable source populations will show less responsivity to variation in the social environment.

In this study we use a field cricket, Teleogryllus oceanicus, to test how social plasticity and sexual trait expression are linked to patterns of genetic divergence. T. oceanicus is widely distributed throughout northern Australia and the Pacific (Otte and Alexander 1983). A previous population genetic study found that the crickets' range historically expanded from west to east, with evidence of successive bottlenecks as founding populations colonised island habitats (Tinghitella et al. 2011), so the system acts as a convenient natural laboratory in which we can study populations with some foreknowledge about their population genetic history (Figure 1). In addition, sexual signals of T. oceanicus are well-characterised, consisting of songs produced by specialised forewing structures, and sex-specific cuticular hydrocarbons (CHCs). Cuticular hydrocarbons are long-chain waxy molecules expressed on the surface cuticle of most insects (Tregenza and Wedell 1997), and CHC profiles appear to be sexually selected in both sexes of $T$. oceanicus (Thomas and Simmons 2009, 2010). 
We tested how $\mathrm{CHC}$ expression is affected by exposure to song. Male calling song is the only known long-range signal by which either sex of this species can detect the abundance and quality of sexually mature males in the local population. $\mathrm{CHC}$ expression in insects is notoriously flexible, and the social environment that males experience is a well-known environmental trigger of such flexibility (e.g. Kent et al. 2008; Chenoweth et al. 2010). CHC profiles in grylline crickets can indicate male qualities such as dominance (Kortet and Hedrick 2005) and attractiveness to females (Thomas and Simmons 2009). Previous work has demonstrated social flexibility in a range of reproductive traits in $T$. oceanicus and allied gryllids, including CHC expression (Bailey et al. 2010; Rebar et al. 2011; Thomas et al. 2011; Bailey and Zuk 2012; DiRienzo et al. 2012; Kasumovic et al. 2012; Tinghitella 2014). Thus, social information in the form of male song is readily available to receivers in the environment, making it a convenient means for manipulating the perception of sexually mature male rivals and testing the impact on a male trait that is known to affect mate choice and other social behaviours with considerable fitness consequences.

We used seven allopatric T. oceanicus populations reared in common-garden conditions to test the relationship between social flexibility in male $\mathrm{CHC}$ profiles and population genetic divergence. We were specifically interested in the idea that social flexibility in short-range $\mathrm{CHC}$ signals could influence the ability of founding individuals to cope with demographic instability. We tested the effects of social experience on $\mathrm{CHC}$ expression by using male calling song playbacks to manipulate the acoustic environment of developing male crickets. The experiment was designed to mimic variation that is likely to be encountered in an initial founding propagule, because an initial colonizing male or males would perceive little to no 
conspecific song in the environment. We estimated the across population variancecovariance matrix (as a measure of genetic divergence) for $\mathrm{CHCs}$ and quantitatively tested whether divergence in $\mathrm{CHC}$ profiles supports an influence of sexual selection in population genetic divergence, above and beyond the action of drift. To evaluate support for the hypothesis that social flexibility is important for individuals in founding populations subject to unpredictable demographic fluctuations, we tested for population-level GSEls in male $\mathrm{CHC}$ expression. We lack the inbred lines necessary to test reaction norm variation of individual genotypes, but show below that $T$. oceanicus populations are genetically differentiated at neutral loci and thus the average genotype is likely to differ among populations. Finally, we tested the prediction that more recently founded populations of $T$. oceanicus exhibit greater social flexibility in male CHC expression.

\section{Methods}

\section{POPULATION ORIGINS AND REARING}

Laboratory populations of $T$. oceanicus were established from seven locations across the species' range: three from Australia, and four from Oceanic islands (Figure 1, Table S1). Populations were derived from offspring of between approximately 20-40 females and males who were housed together for several days. Stock populations were maintained in common garden conditions within a growth chamber at $25^{\circ} \mathrm{C}$ on a photo-reversed $12: 12$ light cycle, following established protocols (e.g. Bailey and Macleod 2014). Adults were reared in 16-L plastic containers at a density of approximately 30-50 individuals, and cleaned twice per week. Crickets were fed ad libitum with Burgess Excel Junior and Dwarf rabbit food and provided moist cotton pads for water and ovipositing and cardboard egg cartons 
for cover. When our protocol required crickets to be individually reared, we isolated them in $118 \mathrm{~mL}$ plastic cups and provided water, rabbit pellets, and cardboard egg carton. We used crickets that had experienced at least two generations of breeding in the lab to mitigate possible field-based maternal effects.

\section{MOLECULAR GENETIC ANALYSIS}

We amplified and scored 10 microsatellite loci in 24 individuals from each population (Tables S1 and S2 in Supporting Information). Six of the loci were previously developed by Beveridge and Simmons (2005): Totri9a, Totri54, Totri55a, Totri57, Totri78, and Totri88a. We developed the remaining four using the program msatcommander (Faircloth 2008) from published T. oceanicus transcriptome data generated using Roche 454 sequencing (Bailey et al. 2013). Candidates were filtered to ensure adequate flanking regions, and primers were designed within those flanking regions. Twenty potential microsatellites were tested and optimised, which yielded four polymorphic loci that we added to the present study: Contig07712, Contig39588, Contig27208, and Contig 12396.

Single hind femurs sampled from wild-caught individuals were preserved in $70 \%$ ethanol. Genomic DNA was extracted using a salt extraction procedure modified from the PureGene protocol (Gentra Systems). Microsatellites were amplified using multiplexing PCR kits (Qiagen) and fluorescently-labelled forward primers following the manufacturer's protocol to a final volume of $10 \mu \mathrm{L}$. The resulting fragments were sequenced on an $A B I 3730$ instrument at Edinburgh Genomics using GeneScan $600 \mathrm{LIZ}$ as a size standard (Applied Biosystems). Allelic identities were scored and checked using Peak Scanner v.1.0 (Applied Biosystems). Details of primer sequences and PCR conditions are supplied in Table S2 in the 
Supporting Information.

GenePop v.4.0.10 (Raymond and Rousset 1995) and FSTAT v.1.2 (Goudet 1995) were used to generate descriptive statistics (number of alleles, observed and expected heterozygosity), test locus-specific Hardy-Weinberg equilibrium, and estimate population-pairwise $F_{S T}$ values. The four microsatellite loci derived from transcriptome sequencing data might be less likely to reflect neutral patterns of genetic divergence than the originally published set of six, which were derived from whole-genome DNA digests. To test this possibility, we compared population-pairwise $\mathrm{F}_{\mathrm{ST}}$ estimates using a paired t-test and assessed the correlation between the two sets of markers. There was potential for pseudoreplication because data from each population appeared more than once in the analyses, so we confirmed results of the latter analysis using a Mantel test with $n=999$ permutations, implemented in the Microsoft Excel add-in GenAIEx v.6.5 (Peakall and Smouse 2012). We found no qualitative difference between patterns of differentiation recovered from each set of loci (see Results) so we proceeded with all analyses using the full set of ten markers. One population (Daintree) deviated significantly from Hardy-Weinberg equilibrium after Bonferroni correction, and one locus (Totri78) showed a similar pattern across all populations after Bonferroni correction (see Results). The latter locus was previously suggested to be susceptible to null alleles (Beveridge and Simmons 2005), but we retained it after verifying that its exclusion did not qualitatively affect estimates of genetic differentiation.

There is debate over the relative merits of different measures of population genetic structure, so we calculated three measures in addition to $F_{S T}$ using GenAIEx v.6.5 (Peakall and Smouse 2012; Verity and Nichols 2014). $F_{S T}^{\prime}, D_{E S T}$ and $G_{S T}^{\prime}$ are standardized measures of page 9 of 65 
genetic differentiation that range from [0,1] (Peakall and Smouse 2012). They may be more suitable for constructing relatedness matrices because $F_{S T}$ can be constrained and never reach the value of 1 , making it difficult to achieve standardized comparisons with other variables (Meirmans and Hedrick 2011). We detected significant genetic differentiation among the majority of populations (see Results). We used Nei's Da (Nei et al. 1983) to visualise this, building a distance matrix with the programme Populations v.1.2.32 (Langella 1999). We used Phylip v.3.695 (Felsenstein 1989) modules "Neighbor" and "Drawtree" to produce an unrooted phylogram (Tinghitella et al. 2011) using the n-Body algorithm, which we re-drew and labelled in Microsoft PowerPoint v.14.0.7159.500.

\section{SONG RECORDING, ANALYSIS, AND PLAYBACK}

Male calling song was recorded for ca. 20 males from each population (Table S1 in the Supporting Information). Adult males were isolated in $118 \mathrm{~mL}$ plastic cups arrayed in a dark room under red light at $25 \pm 2{ }^{\circ} \mathrm{C}$. Recordings were made during the dark phase of the crickets' light:dark cycle using a directional Sennheiser ME66 microphone. Ten complete songs from each recorded individual were manually analysed using Sony Sound Forge 7.0a. For each song, we measured carrier frequency using fast Fourier transform with a 32,768 Blackmann-Harris smoothing window, the durations of sound pulses, and durations of interpulse intervals. Song data from two populations, Daintree and Townsville, have been reported in a previous study (Bailey and Macleod 2014). We used the average call parameters across populations in subsequent acoustic manipulations to ensure playbacks did not favour one population over another.

We constructed 6 artificial playback songs following the method of Brooks et al. (2005). 
Research on a sister species, T. commodus, has highlighted the importance of variation in social cues for triggering socially flexible changes (Kasumovic et al. 2011). Therefore, we designed our acoustic treatment to mimic an environment in which male calling song varied in several key parameters, while the overall mean values and variance for each song trait was held at or close to the average across populations. This also provided a more realistic representation of an acoustic environment likely to be encountered in the field, in which male calling song parameters vary (Simmons et al. 2001). We first manipulated five song traits: (i) carrier frequency, (ii) the number of long chirps, (iii) the long chirp - short chirp interval, (iv) the number of short chirps, and (v) inter-song interval. These were selected on the basis of prior work in T. commodus (Brooks et al. 2005), which estimated multivariate selection acting on song components. Next, we individually adjusted all five parameters of each playback song by multiplying the global standard deviation of each trait by $z$, which determined the number and direction of standard deviations by which to shift each trait value. We calculated $z$ by generating random numbers between $[0,1]$ and obtaining the inverse of the standard normal distribution corresponding to each number using NORMSINV in Microsoft Excel (Microsoft Corp., Redmond, WA) (Brooks et al. 2005). During construction of one song playback (playback 6), the number of long chirps was kept at 5 instead of 7 as was indicated by the above procedure; this was not noted until after data collection was complete. The resulting set of six songs that we selected therefore varied in a largely independent manner for the five traits, yet retained trait means and standard deviations similar to the global values calculated across all populations. Several other traits in our playback songs also varied owing to inherent covariation among some parameters, and we designed the acoustic manipulation below to ensure that crickets from all populations experienced the same acoustic environment to avoid confounding effects. Final values for 
all playback song parameters are given in Table S3 in the Supporting Information.

To construct playbacks with the required trait values, we excised one representative long chirp pulse and one representative short chirp pulse from a laboratory recording, and manually copied and pasted these using Sony Sound Forge 7.0a. Carrier frequency was manipulated using the "pitch adjustment" option in Sony Sound Forge 7.0a, and pulse lengths and inter-pulse intervals were altered where necessary to keep the un-manipulated song parameters as close to constant as possible. To do this, we trimmed pulses to the correct duration and then used the "fade out" option to reshape the sound envelope.

\section{MANIPULATION OF THE ACOUSTIC ENVIRONMENT}

We manipulated the acoustic environment of male crickets during rearing to mimic either a population that has a high density of singing males ("Song" treatment) or a contrasting population that lacked male song ("No Song" treatment). Following previously published protocols (e.g. Bailey and Zuk 2012; Thomas et al. 2011; Kasumovic et al. 2011; Bailey and Macleod 2014) we played back the six artificially-constructed male calling songs on a continuous loop within Jencons LMS Series 4 cooled incubators (Model 600). The temperature was set at $25^{\circ} \mathrm{C}$ and the same $12: 12$ light:dark cycle was used as for stock populations. Individual $118 \mathrm{~mL}$ cricket containers were positioned on trays lined with foam to dampen echoes, above which were suspended Sony SRS-m30 computer speakers attached to CD players (Sony model D-EJ021). Using a CEM-DT 805 sound level meter, we adjusted the sound pressure level of each playback to approximately $80 \mathrm{~dB}$ at the position of the container lids, which simulated, after the acoustic impedance of the lid, a ca. $70 \mathrm{~dB}$ calling song at a cricket's position. Playbacks were timed to coincide with the dark phase of 
the crickets' light:dark cycle. The No Song treatment was similar in all respects except that no song was played back. The use of two social environments precluded investigation of fine-scale shape of the reaction norms recovered, but provided a feasible manipulation with which to estimate and compare reaction norm slopes for our seven populations (Pigliucci 2001).

Four replicate incubators were set up and run simultaneously, with two assigned to each acoustic treatment. For each population, we isolated males and haphazardly assigned them to one of the four incubators when sex differences became apparent. Isolated males were reared in their assigned incubator until adulthood, with food, water, shelter and their position within the incubator changed twice weekly. Upon adult eclosion, we surgically removed the scraper from the left forewing of all crickets; this ensured that males would not sing and disrupt the acoustic treatments. A further 7 - 10 days after eclosion, males were removed from the incubator, flash frozen in their plastic containers for several minutes at $-20{ }^{\circ} \mathrm{C}$, then placed whole into $4 \mathrm{~mL}$ glass vials (QMX Laboratories) and stored at $80^{\circ} \mathrm{C}$.

\section{CHC EXTRACTION AND ANALYSIS}

To extract CHCs, $4 \mathrm{~mL}$ of HPLC-grade hexane (Fisher Scientific) was added to each vial containing a frozen cricket. Crickets were removed after 5 minutes of immersion and the resulting stock extractions were stored at $-20^{\circ} \mathrm{C}$. Subsequently, $100 \mathrm{uL}$ of the extract was aliquoted into $300 \mathrm{uL}$ autosampler vials (Fisher Scientific) and evaporated overnight under a fume hood, leaving only extracted CHCs in the vial. After removal from the hexane wash, each cricket's pronotum length was measured as an estimate of body size. 
$\mathrm{CHC}$ samples were reconstituted in $100 \mathrm{uL}$ of hexane containing an internal standard (10ppm pentadecane). We injected a $2 \mathrm{uL}$ sample of this $\mathrm{CHC}$ extract into a gas chromatography-mass spectrometer (GC-MS; Agilent 7890 GC coupled with an Agilent 5975B mass spectrometer and a CTC PAL autosampler chilled to $5^{\circ} \mathrm{C}$ ) fitted with a DB-WAX column (30 $\mathrm{m} \times 0.25 \mathrm{~mm}$ internal diameter, film $=0.25 \mathrm{um}$ ) using helium as the carrier gas (at a flow rate of $1.1 \mathrm{ml} / \mathrm{min}$ ). We set the inlet temperature to $250^{\circ} \mathrm{C}$ and the injection was conducted in split-less mode. We optimized the separation of the CHC extract using a column profile that started at $50^{\circ} \mathrm{C}$ for 1 minute, then rose at $20^{\circ} \mathrm{C}$ per minute to $250^{\circ} \mathrm{C}$, before holding at this final temperature for 30 minutes (total run time $=41$ minutes). We set the MS transfer line at $230^{\circ} \mathrm{C}$. The electron-impact mass spectra (EI-MS) were recorded with an ionization voltage of $70 \mathrm{eV}$ and a source temperature of $230^{\circ} \mathrm{C} . \mathrm{A} \mathrm{C} \mathrm{C}_{7}-\mathrm{C}_{40}$ straightchained alkane standard was also run in order to calculate retention indices for each $\mathrm{CHC}$ peak (see Table S4 in the Supporting Information).

We calculated the abundance of each CHC peak using MSD CHEMSTATION software (version E.02.00.493; Agilent Technologies) as the area under the peak on the chromatograph (Figure S1 in the Supporting Information). Methyl branched alkanes were identified using Kovat's retention indices and mass spectra (Carlson et al. 1998). Where possible, the position of double bonds in unsaturated compounds was determined by derivitisation with dimethyldisulfide (DMDS) and interpretation of the resulting mass spectra (Francis and Veland, 1981). Two of the peaks were labelled as unidentified, due to their consistently low abundance and the poor quality of their mass spectra. The relative abundance of each peak was measured using ion 57 as the target ion. 


\section{STATISTICAL ANALYSES}

Prior to analysis, we divided the abundance of each $\mathrm{CHC}$ peak by the abundance of the internal standard (pentadecane at $10 \mathrm{ppm}$ ), and the resulting value was $\log _{10}$ transformed (creating a log contrast for each peak) to achieve a normal distribution. Analyses and map construction were performed in R v.2.15.2 (R Core Team, 2012; Becker and Wilks, 2013a and b), SPSS v.21 and ASREML.

Due to the large number of $\mathrm{CHC}$ peaks, we used two multivariate approaches to reduce the dimensionality of our data: Discriminant Function (DF) and Principal Component (PC) analysis. We used DF analysis to visualize the differences in male $\mathrm{CHC}$ expression across populations. We used the Wilks' Lambda $(\lambda)$ statistic to determine which DFs are statistically significant (6 DFs were significant in our analysis) and interpret factor loading greater than $|0.30|$ as biologically significant (Tabachnik and Fidell 1989). Moreover, we use Generalized Cross-Validated (GCV) scores to assess the accuracy of DF analysis to correctly classify our data points according to population. A limitation of this approach is that data can only be separated according to a single factor (i.e. population), which is not compatible with our multifactorial experimental design. We therefore used PC analysis to extract PCs that could be analysed in more complex linear models (Steiger et al. 2013). PCs were extracted from all crickets together (based on the correlation matrix) to ensure that PC scores were directly comparable across levels in our analysis (i.e. populations, social environments). Because we ignored population as a factor when PCs were extracted, the resulting eivenvectors are orthogonal at the phenotypic level, but not necessarily at the genetic level. This enabled us to estimate population-level genetic covariance structure 
based on PCs (Moore 1997). We retained PCs with eigenvalues exceeding 1 for further analysis $(n=6)$ and also interpret factor loadings that exceed $|0.30|$ as biologically important (Tabachnik and Fidell 1989). DF and PC analyses were conducted in IBM SPSS (v.21). We formally tested for and characterised the hypothesised sources of variation in $\mathrm{CHC}$ profiles using a series of linear models. For each of the PCs describing the variation in male $\mathrm{CHC}$ expression we fitted the fixed effect model:

$$
\left.\mathrm{PC}_{\mathrm{n}} \sim \mu+\text { Social }+ \text { Population }+ \text { Social.Population }+ \text { Incubator } \mid \text { Social }+\mathrm{PW}+\varepsilon \text { (model } 1\right)
$$

where $\mu$ is the intercept, "Social" is a two level factor encompassing the social environment (i.e. song vs no song), "Population" is a 7 level fixed factor denoting population of origin and "PW" (pronotum width, mean centred) is included as a linear covariate to control for body size effects. Two replicate incubators were used within each level of the social environment treatment and "Incubator|Social" was therefore included to prevent any bias from incubator effects. $\varepsilon$ represents the random error term.

We then reformulated this as a mixed model with "Population" included as a random rather than a fixed effect. Since "Social.Population" and "Incubator|Social" were never significant under model 1 (see Results) we dropped these to fit the simplified model:

$$
P C_{n} \sim \mu+\text { Social }+P W+\text { Population }+\varepsilon(\text { model } 2)
$$

where "Population" is a random effect and assumed to come from a normal distribution with a mean of zero and variance to be estimated $\left(V_{P O P}\right)$. PC scores were modelled in 
standard deviation units such that $\mathrm{V}_{\mathrm{POP}}$ is interpretable as the proportion of phenotypic variance due to genetic differences among populations. This simple mixed model was then extended in two ways. First, we extended it to the multivariate case to estimate the full among population genetic variance-covariance matrix among PCs (Model 3). Second, for each of the six PCs we partitioned $\mathrm{V}_{\mathrm{POP}}$ into components comprising (i) genetic variance consistent with divergence under drift alone ( $\mathrm{V}_{\text {POPdrift }}$ ), and (ii) additional genetic variance (beyond neutral expectations) that would be indicative of divergent selection ( $\left.\mathrm{V}_{\mathrm{POPsel}}\right)$. To do this we added an additional random effect such that:

$$
\mathrm{PC}_{\mathrm{n}} \sim \mu+\text { Social }+\mathrm{PW}+\text { Pop }_{\text {drift }}+\text { Pop }_{\mathrm{sel}}+\varepsilon \quad(\text { Model } 4)
$$

where we assume that the expected genetic covariance between any pair of observations on populations $\left(\mathrm{Pop}_{\mathrm{i}, \mathrm{j}}\right.$ ) under a neutral model of among-population divergence equal to (1$\mathrm{F}_{S T \mathrm{ij}}^{\prime}$ ) is $\mathrm{V}_{\mathrm{POPdrift}}$ (where $\mathrm{F}_{S T}^{\prime}$ is the microsatellite based measure of genetic distance scaling from 0 to 1 as described above). The second random effect of "Pop sel" is modelled identically to the simple random effect of population under model 2 to estimate $V_{\text {Popsel. }}$. To test whether genetic variation among populations was greater than expected under drift

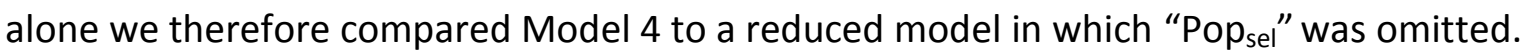
All linear models were fitted using restricted maximum likelihood with conditional $F$ tests used for inference on fixed effects. We used likelihood ratio tests (LRT) for inference on random effects but caution that the asymptotic approximation of this test statistic to a $\chi^{2}$ distribution may not give reliable results with only seven levels of the random effect (i.e. distinct populations). We therefore provide $P$ values but stress they should be interpreted cautiously. 
We performed a post-hoc analysis upon detecting an interesting pattern in our population genetic results (below), which suggested a genetic break between mainland and island populations with a corresponding decline in allelic richness (Figure 1). To test whether social flexibility was related to qualitative differences between mainland vs. island populations, as opposed to finer-scale quantitative differences reflecting colonisation history, we expanded model 1 with additional terms to include land type effects. These specified whether populations were derived from mainland Australia or islands (Landtype), accounted for among-population variation by nesting Population within Landtype (Population | Landtype), and tested whether mainland and island populations showed different reaction norm slopes (Social.Landtype) or whether populations within a given land type responded differently to the social manipulation (Social.Population|Landtype):

$$
\begin{array}{r}
\mathrm{PC}_{\mathrm{n}} \sim \mu+\text { Social }+ \text { Landtype }+ \text { Social.Landtype }+ \text { Incubator } \mid \text { Social }+ \text { Population | Landtype }+ \\
\text { Social.Population |Landtype }+\mathrm{PW}+\varepsilon \quad(\text { model } 5)
\end{array}
$$

\section{Results}

\section{POPULATION GENETICS}

Populations were genetically differentiated, with all but two pairwise $\mathrm{F}_{\mathrm{ST}}$ comparisons statistically significant after Bonferroni correction $(\alpha=0.0024)$ (Table 1). Patterns of differentiation calculated using the methods of $F^{\prime}{ }_{S T}, G^{\prime}{ }_{S T}$, and $D_{E S T}$ yielded qualitatively similar results (Table 1). Estimates of $\mathrm{F}_{\mathrm{ST}}$ derived from the previously published markers and our transcriptome-based markers were not different (paired t-test: $N=21, t=0.28, P=$ 
0.781), and population-pairwise distance estimates were positively correlated among the two sets of loci (Pearson correlation: $N=21, r=0.665, P=0.001$; Mantel test: $N=21, \mathrm{r}_{\mathrm{xy}}=$ $0.665, P=0.002)$.

Allele numbers for the ten loci ranged between 7 and 35 and are detailed in Table S2 in the Supporting Information. In contrast to Beveridge and Simmons (2005), we found no pattern of heterozygosity deficit consistent with X-linkage at Totri88a: observed global heterozygosity of 0.636 did not depart significantly from the expectation of $0.617(P=$ 0.496), and when examined by population, it only deviated from Hardy-Weinberg equilibrium (HWE) in Daintree $(P<0.001)$. When analysed separately by sex, Daintree remained the only population showing a significant male heterozygote deficit $(P<0.001)$; all remaining sexes and populations were in HWE at Totri88a (all $P \geq 0.269$ ). Patterns of genetic differentiation across Australia and Oceania were consistent with Tinghitella et al. (2011) and suggest a phylogeographic break corresponding approximately to populations in the Polynesian Triangle (Figure 1b). Mean allelic diversity (Figure 1c) confirmed this pattern, and was consistent with the progressive loss of alleles in more easterly populations found by Tinghitella et al. (2011). However, with the exception of Fiji, allelic diversity was largely similar among mainland Australian populations, and among Oceanic populations, suggesting a biologically relevant distinction among mainland vs. island populations.

\section{POPULATION DIVERGENCE IN MALE CHC PROFILES}

GC-MS analysis of male $\mathrm{CHC}$ extracts revealed 26 individual $\mathrm{CHCs}$ ranging in chain length from $\mathrm{C}_{28}$ to $\mathrm{C}_{33}$ and consisting of a mixture of methylalkanes, dimethylalkanes, alkenes and alkadienes (Table S4, Figure S1 in the Supporting Information). PC analysis of these 26 CHC 
peaks yielded 6 PCs with eigenvalues exceeding 1, which collectively explain $75.85 \%$ of the total variation in male $\mathrm{CHC}$ expression. Table S5 in the Supporting Information provides eigenvalues and factor loading scores for the 6 principal components associated with $\mathrm{CHC}$ variation. PC1 accounts for $38.40 \%$ of the variance in male $\mathrm{CHC}$ expression and is positively loaded to each $\mathrm{CHC}$ peak. Consequently, this vector describes the absolute amount of $\mathrm{CHCs}$ produced by a male. PC2 explained a further $16.53 \%$ of the variation in male $\mathrm{CHC}$ expression and was positively loaded to 8 peaks (peaks $17-21$ and 24-26) and negatively loaded to 5 peaks (peaks 11 and 13-16). Based on the retention times of these peaks (Figure S1 in the Supporting Information), this vector describes the trade-off between long and short-chained CHCs. PC3 explained a further $7.28 \%$ of the variation in male $\mathrm{CHC}$ expression and was positively loaded to 5 peaks (peaks 1, 6, 10, 12 and 13) and negatively loaded to 3 peaks (peaks 5, 20 and 25) and therefore also describes the trade-off between specific CHCs but is unrelated to chain length. PC4 explained a further $5.14 \%$ of the variation in male $\mathrm{CHC}$ expression and is positively loaded to 2 peaks (peaks 22 and 23) and negatively loaded to 2 peaks (peaks 12 and 13). Thus, this vector is similar to PC2 in describing the trade-off between long and short-chained CHCs. PC5 explains a further $4.56 \%$ of the variance in male CHC expression and is positively loaded to 4 peaks (peaks 1, 8, 9 and 22) and negatively loaded to 2 peaks (peaks 10 and 25). Consequently this vector is similar to PC3 in describing the trade-off between specific $\mathrm{CHCs}$ that are unrelated to chain length. Finally, PC6 explains the remaining $3.92 \%$ of the variation in male $\mathrm{CHC}$ expression and is positively loaded to 3 peaks (peaks 9, 21 and 22) and therefore this vector represents an increased expression of these specific CHCs. 
Model 1 provides evidence for significant population differences in all PCs that describe the variation in male $\mathrm{CHC}$ expression (Table 2). This population divergence in male $\mathrm{CHC}$ expression can be clearly visualized along the first two DFs (Figure 2) and shows a close resemblance to the difference in allelic frequencies observed across populations (Figure 1c). In total, 17 out of the $26 \mathrm{CHC}$ peaks contributed to one or both of these DFs (Table S6 in the Supporting Information) and the analysis correctly classified $63.2 \%$ of cross-validated grouped cases. The success of this analysis, however, varied substantially across populations with GCV scores being lower for the three Australian populations (Daintree $=47.4 \%$, Mackay $=52.8 \%$ and Townsville $=51.6 \%)$ than the four Oceanic populations $(\mathrm{Fiji}=72.2 \%$, Mangaia $=$ $72.5 \%$, Tahiti $=75.9 \%$ and Hilo $=66.1 \%)$ indicating that the former populations could not be as accurately classified as the latter populations.

\section{SOCIAL EFFECTS ON MALE CHC PROFILES}

Social environment effects were also found for PC1 and PC3 and approached significance for PC4 (Table 2). Collectively, this demonstrates that male CHC expression is influenced by both genetic and social environment effects. However, we found no statistical support for Social.Population effects and therefore no evidence for genotype by (social) environment interaction at the among-population level. Thus, while the mean expression of CHCs differs across populations, and to a lesser degree across social environments, there was no difference in the slopes of the reaction norms describing how each population responds to the two social environments examined here (Figure 3). PC1, PC2 and PC6 all increased significantly with male body size (pronotum width), and there was no evidence of incubator effects within each treatment (Table 2). 
Post-hoc analyses uncovered minimal evidence that males from mainland Australian vs. island populations modified their CHC expression differently depending on the prevailing acoustic environment (Table S7). The critical Landtype.Social interaction term only marginally approached significance for PC2 and PC6 (GLMs for PC2 and PC6, respectively: $\left.F_{3,751}=3.83, P=0.052 ; F_{3,751}=3.88, P=0.051\right)$. These PCs explained $16.5 \%$ and $3.9 \%$ of total CHC variance, respectively. For PC2, which explained the greater amount of variance, the estimated average effect of experiencing the song environment reversed direction in mainland vs. island populations, as indicated by coefficients of 0.102 vs. -0.139 . Though this reversal was not significant, it illustrates that the absolute magnitude of social flexibility described by PC2 did not differ among mainland or island populations, though the direction of the response did.

\section{RELATIONSHIP BETWEEN CHC PROFILES AND GENETIC DIVERGENCE}

Univariate formulations of model 2 confirmed these findings and yielded estimates of $V_{P O P}$ that ranged from $10-40 \%$ of the total phenotypic variance across populations and were all nominally significant based on LRTs (see Table S8 in the Supporting Information). Under the multivariate formulation (model 3), inclusion of population covariance among the PCs describing variation in male $\mathrm{CHC}$ profiles significantly improved the model $\left(\chi_{21}^{2}=31.74, P=\right.$ 0.007) such that we conclude there is evidence for genetic covariance among, as well as variance in, individual $\mathrm{CHC}$ traits. The corresponding genetic correlation estimates (as well as $V_{\text {POP }}$ scaled by $V_{P}$ ) from the 6 trait model are shown in Table 3.

However, while it is clear that there is genetic (co)variation among populations in the PCs that describe the variation in male $\mathrm{CHCs}$, we found no evidence for significantly greater 
divergence than expected under a neutral model (Table 4). In four of the six traits the estimate of $V_{\text {POPsel }}$ was bound to zero, while divergence by drift explains $67 \%$ and $95 \%$ of genetic variation in PC1 and PC5, respectively.

\section{Discussion}

Decades of research have emphasized the importance of sexual selection in the elaboration of sexual ornaments and preferences (Andersson and Simmons 2006), and it is widely suggested to be a potent accelerator of evolutionary change (Fisher 1958; Lande 1981; Mendelson and Shaw 2005). Nevertheless, researchers have not reached consensus on whether and in what circumstances sexual selection contributes causally to the development of reproductive isolation, thereby "driving" evolutionary diversification (Ritchie 2007; Scordato et al. 2014; Rodríguez et al. 2013a; Safran et al. 2013). The core of the debate centres on whether sexual selection itself is a diversifying source of selection on ornaments and preferences in different populations, or whether sexual selection is more akin to a background process that translates ecological selection or drift into variation in reproductively isolating traits (Ritchie 2007). Under the latter scenario, sexual selection need not be the causative selective force driving diversification of sexual traits and preferences. Instead, it might act to exaggerate sexual traits in a direction constrained by ecological or biotic factors, as has been illustrated in a recent study of the treehopper Enchenopa binotata (Rebar and Rodríguez 2015). This is more than just a semantic argument: the architects of the modern synthesis recognised that it is necessary to partition the potentially overlapping effects of stochastic processes such as founder events and drift from different forms of selection to understand evolutionary diversification (Coyne and Orr 
2004).

The fact that sexually selected trait expression may also be flexible depending on the social environment adds further complications to evaluating the role of sexual selection in divergence (Cornwallis and Uller 2010). However, it also provides a testable prediction: if social effects on expression of sexually selected traits vary in different populations, the ensuing evolutionary dynamics generated by feedback arising from the social environment could push trait evolution in different directions in different populations (Bailey and Moore 2012; Rebar and Rodríguez 2013, 2015; Wolf et al. 2014). Across multiple allopatric populations of $T$. oceanicus, we found abundant evidence for variation in a male trait suspected to be under sexual selection - CHC profiles. In addition, accumulating evidence suggests $\mathrm{CHC}$ profiles respond with particular sensitivity to cues or signals in the social environment (Kent et al. 2008; Thomas et al. 2011; Gershman et al. 2014), and consistent with this, we found that exposure to acoustic signals during rearing in the form of male calling songs also modulated the expression of some combinations of male CHCs. Nevertheless, we did not detect evidence of population-level gene by (social) environment interactions in $\mathrm{CHC}$ expression. Male crickets from populations across a wide portion of the T. oceanicus range responded in a consistent manner to the presence or absence of calling songs in their rearing environment.

Gene by (social) environment interactions ensue when social effects are not homogeneous among populations that are genetically divergent, and such GSEls are predicted to contribute to different responses to selection for the traits involved (Wolf et al. 2014). However, this appears not to be the case for male $\mathrm{CHC}$ profiles in the cricket populations we 
studied. Reaction norms describing social effects on multivariate principal components did depart significantly from a slope of zero, yet the slope and magnitude of reaction norms was similar across populations. Our analysis is necessarily limited by the fact that we were unable to sample individual genotypes. For instance, GSEls might occur within a population, but when estimated at the population level could be obscured owing to the effects of averaging across numerous sampled genotypes (Pigliucci 2001). Nevertheless, we found no evidence that social flexibility differed among $T$. oceanicus populations. This lack of population-level GSEls stands in contrast to a prior study that described variation in indirect genetic effects in female choosiness in the same species (Bailey and Zuk 2012). Although GSEls and indirect genetic effects are not equivalent-because genetic variation is not required to underlie different social environments contributing to GSEls - variation among populations in the strength and/or direction of indirect genetic effects would imply GSEls. The latter study estimated variation among five populations in the parameter $\psi$, which describes the magnitude and direction of indirect genetic effects arising from the social environment. Significant variation in $\psi$ suggests GSEls, and despite the clear differences between the two studies (different populations, sexes and traits), the reasons why some apparently labile traits exhibit GSEls and why others do not remains an open question. Chenoweth et al. (2010) showed that applying experimental sexual selection pressure to populations of Drosophila serrata could result in the evolution of indirect genetic effects on CHC expression in this species. The $D$. serrata findings illustrate that reaction norms describing responses to social environments need not be fixed, and if genetic variation exists for reaction norm shape, we might similarly expect GSEls to evolve over time.

There were compelling reasons to consider that mainland and island populations of $T$. 
oceanicus might show different dynamics of $\mathrm{CHC}$ expression and social flexibility, including an apparent phylogeographic break around the Polynesian Triangle and lower allelic diversity in island populations (Figure 1). These patterns contrasted counterintuitively with the higher variability in $\mathrm{CHC}$ expression we found in island populations (Figure 2). Historical biogeography drives unusual sexual selection dynamics in many systems, for example the lizard Podarcis gaigeae (Runemark et al. 2011), and numerous arthropods (Gillespie and Roderick 2002). In T. oceanicus, asymmetric female discrimination of song-deficient males is correlated with the putative colonisation history of populations across the species range (Tinghitella and Zuk 2009). However, we did not find an unambiguous difference between mainland and island populations in socially flexible $\mathrm{CHC}$ expression: average $\mathrm{CHC}$ profiles differed between the land types, but mainland and island populations responded similarly to acoustic experience. The interaction between land type and social experience approached significance at the $\alpha=0.05$ level for two principal components describing $\mathrm{CHC}$ variation, but the effect appeared to be weak. A possible explanation for this is that social flexibility in $\mathrm{CHC}$ expression has important fitness consequences that are relatively consistent across ecological contexts. Its additional functions in establishing dominance and modulating aggressive encounters (Kortet and Hedrick 2005) may place constraints on the evolution of plasticity. Interactions among signal modalities are increasingly recognised to affect the dynamics of sexual selection (Reichert and Höbel 2015), and it would be beneficial to consider whether we predict similar degrees and directions of social flexibility for signalling traits in different modalities and channels.

Our experimental design enabled us to estimate an among population genetic covariance matrix for components of $\mathrm{CHC}$ profiles in male $T$. oceanicus. The estimates revealed modest 
genetic variance for the principal components that describe $\mathrm{CHC}$ variation, but generally low genetic covariances among these principal components. In our analysis, we extracted PCs at the phenotypic, not the genetic, level (Kirkpatrick and Meyer 2004). While in general PC axes are not expected to show phenotypic covariation owing to their orthogonal construction, genetic covariance among PC axes can nonetheless be estimated (Moore 1997). An absence of genetic covariance among PCs does not imply absence of genetic covariance among $\mathrm{CHCs}$, as the PCs are themselves composites of individual CHC traits. However, the apparently negligible genetic integration revealed in the population genetic covariance matrix might help explain why not all PCs showed evidence of social flexibility in response to the acoustic environment. If different axes of variation in male $\mathrm{CHC}$ expression are coordinated by loci that tend not to be in linkage disequilibrium, selection on male $\mathrm{CHC}$ expression may be relatively unconstrained and plasticity in one component need not correlate with plasticity in another. In insect species where $\mathrm{CHC}$ variation has been genetically mapped, perhaps unsurprisingly, phenotypic variation tends to localise to many quantitative trait loci (e.g. Niehuis et al. 2011). The best studied are drosophilids, in which a number of desaturase and elongase genes have been identified as affecting the synthesis and expression of $\mathrm{CHCs}$ (Howard and Blomquist 2005). However, there is evidence for substantial genetic decoupling of CHC blends in D. melanogaster (Foley et al. 2007), consistent with our finding of low genetic covariance between the PCs describing CHC variation across populations.

Having found considerable variation in $\mathrm{CHC}$ expression across populations due to a combination of genes and the social environment (but not GSEIs), there remains one piece of evidence addressing our hypothesis about the role of male CHCs in population 
divergence. That is our ability to explain population variation in $\mathrm{CHCs}$ using models that compare the predictive power of drift, derived from an analysis of putatively neutral loci, versus drift plus the action of selection. Again we found no evidence to support the idea that sexual selection has shaped population variation in male $\mathrm{CHC}$ profiles. Assuming that the microsatellite loci used in this study are selectively neutral, levels of $\mathrm{CHC}$ variation observed among populations are not greater than might be expected under drift alone. The pattern of allelic drop out across mainland Australia and successive Oceanic island populations indicates eastward expansion with successive bottlenecks in founding populations, a situation in which drift is expected to exert a powerful influence on the genetic and phenotypic composition of populations. We acknowledge that our analysis may not have been refined enough to detect subtle influences of sexual selection on $\mathrm{CHC}$ divergence above and beyond strong effects of drift in a small number of island populations, and indeed other forms of selection may also act on $\mathrm{CHC}$ profiles. Insect $\mathrm{CHCs}$ have a known function in dessication resistance, and although $T$. oceanicus populations are found in similar environments across their tropical range, microhabitat differences might exert selection on $\mathrm{CHC}$ composition. However if that were the case, we would expect to detect signatures of selection above and beyond those indicated in the drift-only model. Sexual selection might also oppose natural selection on $\mathrm{CHCs}$, such that net phenotypic variation is minimal, but this is a less parsimonious scenario. Finally, it is also possible that sexual selection is indeed imposed on CHCs but that its impact on $\mathrm{CHC}$ divergence is uniform. In other words, sexual selection might simply accentuate the signature of genetic drift such that it is less likely to be detected in our analysis. However, despite these caveats, our null result stands in contrast to studies documenting sexual selection on $\mathrm{CHC}$ profiles in insects, for example in the sagebrush cricket Cyphoderris strepitans (Steiger et al. 2013), Drosophila 
montana (Veltsos et al. 2008), and the species studied here, T. oceanicus (Thomas and Simmons 2009; 2010).

\section{Conclusions}

Male CHC expression in T. oceanicus clearly varies across populations and is sensitive to acoustic cues in the environment. However, we found no evidence that $\mathrm{CHC}$ elaboration through sexual selection-or natural selection-has played a large role in population divergence, either through GSEls imposed by variation in the acoustic environment of founding populations, or by rapid phenotypic evolution in different directions. We cannot definitively exclude the idea that ecological selection acts on $\mathrm{CHC}$ profiles differently across the $T$. oceanicus range we sampled, and that the genetic markers and $\mathrm{CHC}$ markers we assayed both reflect this. However, two lines of evidence fail to implicate sexual selection as a driving force in patterns of population differentiation, in favour of a scenario in which $\mathrm{CHC}$ profiles vary across the range of $T$. oceanicus in a pattern underpinned by random drift.

The role of GSEIs (or, when genetic variation influences the social environment, variation in the interaction coefficient $\psi$ ) in impeding or promoting evolutionary diversification remains to be empirically tested. An outstanding question is whether GSEIs are more likely to be found for traits that are unusually susceptible to variation in the social environment. The prediction under such a scenario would be a positive association with the magnitude of social flexibility and the presence of GSEls. It is possible, as with the field cricket examples elaborated above, that behaviour is inherently more labile and reversible than traits that become fixed during development, such as many morphological features, although counter- 
examples suggest a need to test this (e.g. Chaine and Lyon 2008). Flexibility in mating signals and preferences is a burgeoning area of research (Rodríguez et al. 2013b), and the dynamism of sexual selection pressures even across relatively small spatial scales is becoming increasingly appreciated (Gosden and Svensson 2008). Future efforts to delimit causal relationships between traits elaborated under sexual selection and population divergence would benefit from testing more explicit predictions about the social flexibility of different types of traits, and how different manifestations of that social flexibility, e.g. its magnitude, the presence of GSEIs, or involvement of IGEs, contributes to broader patterns of diversification.

\section{ACKNOWLEDGEMENTS}

We thank David Forbes and Audrey Grant for cricket maintenance. Paris Veltsos provided valuable support for developing new microsatellite loci, as did the Sanger Sequencing Centre at the Edinburgh Genomics Institute. We are grateful to Benjamin Freeman for helping to extract DNA. William Bailey, Stephen Blanksby, Brian Gray, Simon Hodge, Glenda Jones, Rhedyn Ollerynshaw, John Rotenberry, Suzanne Vardy and Marlene Zuk greatly assisted with field collections. Michael Ritchie, Erik Svensson and two anonymous reviewers and the editor provided helpful feedback on earlier versions of the manuscript. N.W.B. was funded by Natural Environment Research Council (NERC) fellowships and support for S.P. was provided by a NERC Standard Grant (NE/G014906/1, NE/L011255/1, NE/I027800/1). A University of California Pacific Rim Research Grant (08.T.PRRP.05.0029) provided additional funding. J.H. received support from a University Royal Society Fellowship and Royal Society Equipment Grant, A.J.W. from a BBSRC David Phillips Fellowship, and M.M. was supported 
by an Erasmus exchange programme grant. The authors declare no conflicts of interest.

\section{DATA ARCHIVING}

Data is archived on Dryad at doi:10.5061/dryad.tb552.

\section{LITERATURE CITED}

Agrawal, A. F., E. D. Brodie III, and M. J. Wade. 2001. On indirect genetic effects in structured populations. The American Naturalist 158:308-323.

Andersson, M., and L. W. Simmons. 2006. Sexual selection and mate choice. Trends in Ecology and Evolution 21:296-302.

Bailey, N. W. 2012. Evolutionary models of extended phenotypes. Trends in Ecology and Evolution 27:561-569.

Bailey, N. W., and E. Macleod. 2014. Socially flexible female choice and premating isolation in field crickets (Teleogryllus spp.). Journal of Evolutionary Biology 27:170-180.

Bailey, N. W., and A. J. Moore. 2012. Runaway sexual selection without genetic correlations: social environments and flexible mate choice initiate and enhance the Fisher process. Evolution 66:2674-2684. 
Bailey, N. W., P. Veltsos, Y. -F. Tan, A. H. Millar, M. G. Ritchie, and L. W. Simmons. 2013. Tissue-specific transcriptomics in the field cricket Teleogryllus oceanicus. Genes, Genomes, Genetics 3:225-230.

Bailey, N. W., and M. Zuk. 2012. Socially flexible female choice differs among populations of the Pacific field cricket: geographic variation in the interaction coefficient psi ( $\psi)$. Proceedings of the Royal Society of London, B 279:3589-3596.

Baldwin, J. M. 1896. A new factor in evolution. The American Naturalist 30:441-451, 536553.

Becker, R. A., and A. R. Wilks. 2013a. mapdata: Extra Map Databases. R version by Ray Brownrigg. Enhancements by Thomas P Minka. http://CRAN.Rproject.org/package=mapdata .

Becker, R. A., and A. R. Wilks. 2013b. maps: Draw Geographical Maps. R version by Ray Brownrigg. Enhancements by Thomas P Minka. http://CRAN.Rproject.org/package=maps .

Beveridge, M., and L. W. Simmons. 2005. Microsatellite loci for the Australian field cricket Teleogryllus oceanicus and their cross-utility in Teleogryllus commodus. Molecular Ecology Notes 5:733-735.

Bijma, P. 2014. The quantitative genetics of indirect genetic effects: a selective review of Pascoal et al. Divergence and Social Flexibility page 32 of 65 
modelling issues. Heredity 112:61-69.

Brooks, R., J. Hunt, M. W. Blows, M. J. Smith, L. F. Bussière, and M. D. Jennions. 2005. Experimental evidence for multivariate stabilizing sexual selection. Evolution 59:871880.

Carlson, D.A., U.R. Bernier, and B.D. Sutton. 1998. Elution patterns from capillary GC for methyl-branched alkanes. Journal of Chemical Ecology 24: 1845-1865.

Chaine, A. S., and B. E. Lyon. 2008. Adaptive plasticity in female mate choice dampens sexual selection on male ornaments in the lark bunting. Science 319:459-462.

Chenoweth, S. F., H. D. Rundle, and M. W. Blows. 2010. Experimental evidence for the evolution of indirect genetic effects: changes in the interaction coefficient, psi $(\Psi)$, due to sexual selection. Evolution 64:1849-1856.

Cornwallis, C. K., and T. Uller. 2010. Towards an evolutionary ecology of sexual traits. Trends in Ecology and Evolution 25:145-152.

Coyne, J. A., and H. A. Orr. 2004. Speciation. Sinauer, Sunderland, USA.

DeWitt, T. J., A. Sih, and D. S. Wilson. 1998. Costs and limits of plasticity. Trends in Ecology and Evolution 13:77-81. 
DiRienzo, N., J. N. Pruitt, and A. V. Hedrick. 2012. Juvenile exposure to acoustic signals from conspecifics alters growth trajectory and an adult personality trait. Animal Behaviour 84:861-868.

Dukas, R. 2008. Evolutionary biology of insect learning. Annual Reviews in Entomology 53:145-160.

Faircloth, B. C. 2008. msatcommander: detection of microsatellite repeat arrays and automated, locus-specific primer design. Molecular Ecology Resources 8:92-94.

Felsenstein, J. 1989. PHYLIP - Phylogeny Inference Package (Version 3.2). Cladistics 5:164166.

Foley, B., S. F. Chenoweth, S. V. Nuzhdin, and M. W. Blows. 2007. Natural genetic variation in cuticular hydrocarbon expression in male and female Drosophila melanogaster. Genetics 175:1465-1477.

Francis, G.W., and K. Veland. 1981. Alkylthiolation for the determination of double-bond positions in linear alkenes. Journal of Chromotography 219: 379-384.

Gershman, S. N., E. Toumishey, and H. D. Rundle. 2014. Time flies: Time of day and social environment affect cuticular hydrocarbon sexual displays in Drosophila serrata. Proceedings of the Royal Society of London, Series B. 281:40821 
Gosden, T. P., and E. I. Svensson. 2008. Spatial and temporal dynamics in a sexual selection mosaic. Evolution 62:845-856.

Goudet, J. 1995. FSTAT (version 1.2) A computer program to calculate F-statistics. Journal of Heredity 86:385-386.

Hunt, J., R. R. Snook, C. Mitchell, H. S. Crudgington, and A. J. Moore. 2012. Sexual selection and experimental evolution of chemical signals in Drosophila pseudoobscura. Journal of Evolutionary Biology 25:2232-2241.

Kasumovic, M. M., M. D. Hall, and R. C. Brooks. 2012. The juvenile social environment introduces variation in the choice and expression of sexually selected traits. Ecology and Evolution 2:1036-1047.

Kasumovic, M. M., and R. C. Brooks. 2011. It's all who you know: the evolution of socially cued anticipatory plasticity as a mating strategy. The Quarterly Review of Biology 86:181-197.

Kasumovic, M. M., M. D. Hall, H. Try, and R. C. Brooks. 2011. The importance of listening: juvenile allocation shifts in response to acoustic cues of the social environment. Journal of Evolutionary Biology 24:1325-1334.

Kent, C., R. Azanchi, B. Smith, A. Formosa, and J. D. Levine. 2008. Social context influences chemical communication in D. melanogaster males. Current Biology 18:1384-1389. 
Kirkpatrick, M., and K. Meyer. 2004. Direct estimation of genetic principal components: simplified analysis of complex phenotypes. Genetics 168:2295-2306.

Kortet, R., and A. Hedrick. 2005. The scent of dominance: female field crickets use odour to predict the outcome of male competition. Behavioural Ecology and Sociobiology 59:77-83.

Lande, R. 1981. Models of speciation by sexual selection on polygenic traits. Proceedings of the National Academy of Sciences, USA 78:3721-3725.

Langella, O. 1999. Populations 1.2.32. Population genetic software. Laboratoire Evolution, Génomes et Spéciation, CNRS UPR9034, Gif-sur-Yvette (France).

Majlát, P., Z. Erdos, and J. Takacs. 1974. Calculation and application of retention indices in programmed-temperature gas-chromatography. Journal of Chromatography 91:89103.

Meirmans, P. G., and P. W. Hedrick. 2011. Assessing population structure: $F_{S T}$ and related measures. Molecular Ecology Resources 11:5-18.

Mendelson, T. C., and K. L. Shaw. 2005. Rapid speciation in an arthropod. Nature 433:375376. 
Miller, C. W., and E. I. Svensson. 2014. Sexual selection in complex environments. Annual Review of Entomology 59:427-445.

Moore, A. J. 1997. The evolution of sexual signals: morphological, functional, and genetic integration of the sex pheromone in Nauphoeta cinerea. Evolution 51:1920-1928.

Moore, A. J., E. D. Brodie III, and J. B. Wolf. 1997. Interacting phenotypes and the evolutionary process: I. Direct and indirect genetic effects of social interactions. Evolution 51:1352-1362.

Moore, A. J., and T. Pizzari. 2005. Quantitative genetic models of sexual conflict based on interacting phenotypes. The American Naturalist 165:S88-S97.

Nei, M., F. Tajima, and Y. Tateno. 1983. Accuracy of estimated phylogenetic trees from molecular data. 2. Gene-frequency data. Journal of Molecular Evolution 19:153-170.

Niehuis, O., J. Büllesbach, A. K. Judson, T. Schmitt, and J. Gadau. 2011. Genetics of cuticular hydrocarbon differences between males of the parasitoid wasps Nasonia giraulti and Nasonia vitripennis. Heredity 107:61-70.

Oh, K. P., G. L. Conte, and K. L. Shaw. 2013. Founder effects and the evolution of asymmetrical sexual isolation in a rapidly-speciating clade. Current Zoology 59:230238. 
Otte, D., and R. D. Alexander. 1983. The Australian crickets (Orthoptera: Grillidae). Academy of Natural Sciences of Philadelphia. Philadelphia, PA.

Peakall, R., and P. E. Smouse. 2012. GenAlEx 6.5: genetic analysis in Excel. Population genetic software for teaching and research -an update. Bioinformatics 28:25372539.

Petfield, D., S. F. Chenoweth, H. D. Rundle, and M. W. Blows. 2005. Genetic variance in female condition predicts indirect genetic variance in male sexual display traits. Proceedings of the National Academy of Sciences, U.S.A. 102:6045-6050.

Pigliucci, M. 2001. Phenotypic plasticity: beyond nature and nurture. The Johns Hopkins University Press.

R Core Team. 2012. R: A language and environment for statistical computing. R Foundation for Statistical Computing, Vienna, Austria. http://www.R-project.org/.

Raymond, M., and F. Rousset. 1995. GENEPOP (version 1.2): population genetics software for exact tests and ecumenicism. Journal of Heredity 86:248-249.

Rebar, D., and R. L. Rodríguez. 2013. Genetic variation in social influence on mate preferences. Proceedings of the Royal Society of London, B 280:1-7.

Rebar, D., and R. L. Rodríguez. 2015. Insect mating signal and mate preference phenotypes Pascoal et al. Divergence and Social Flexibility page 38 of 65 
covary among host plant genotypes. Evolution 69:602-610.

Rebar, D., M. Zuk, and N. W. Bailey. 2011. Mating experience in field crickets modifies preand post-copulatory female choice in parallel. Behavioral Ecology 22:303-309.

Reichert, R. S., and G. Höbel. 2015. Modality interactions alter the shape of acoustic mate preference functions in gray treefrogs. Evolution doi:10.1111/evo.12750

Rhodes, S. B., and I. Schlupp. 2012. Rapid and socially induced change of a badge of status. Journal of Fish Biology 80:722-727.

Ritchie, M. G. 2007. Sexual selection and speciation. Annual Reviews in Ecology and Systematics 38:79-102.

Rodríguez, R. L., J. W. Boughman, D. A. Gray, E. A. Hebets, G. Höbel, and L. B. Symes. $2013 a$. Diversification under sexual selection: the relative roles of mate preference strength and the degree of divergence in mate preferences. Ecology Letters 16:964-974.

Rodríguez, R. L., D. Rebar, K. D. Fowler-Finn. 2013b. The evolution and evolutionary consequences of social plasticity in mate preferences. Animal Behaviour 85:10411047.

Rousset, F. 2008. Genepop'007: a complete reimplementation of the Genepop software for Windows and Linux. Molecular Ecology Resources 8:103-106. 
Safran, R. J., E. S. C. Scordato, L. B. Symes, R. L. Rodríguez, and T. C. Mendelson. 2013. Contributions of natural and sexual selection to the evolution of premating reproductive isolation: a research agenda. Trends in Ecology and Evolution 28:643650.

Scordato, E. S. C., L. B. Symes, T. C. Mendelson, and R. J. Safran. 2014. The role of ecology in speciation by sexual selection: a systematic empirical review. Journal of Heredity 105:782-794.

Servedio, M. R., S. A. Sæther, and G.-P. Sætre. 2009. Reinforcement and learning. Evolutionary Ecology 23:109-123.

Simmons, L. W., M. Zuk, J. T. Rotenberry. 2001. Geographic variation in female preference functions and male songs of the field cricket Teleogryllus oceanicus. Evolution $55: 1386-1394$.

Steiger, S., G. D. Ower, J. Stökl, C. Mitchell, J. Hunt, and S. K. Sakaluk. 2013. Sexual selection on cuticular hydrocarbons of male sagebrush crickets in the wild. Proceedings of the Royal Society of London, Series B. 280:20132353.

Svensson, E. I., F. Eroukhmanoff, K. Karlsson, A. Runemark, and A. Brodin. 2010. A role for learning in population divergence of mate preferences. Evolution 64:3101-3113. 
Szúcs, M., B. A. Melbourne, T. Tuff, and R. A. Hufbauer. 2014. The role of demography and genetics in the early stages of colonization. Proceedings of the Royal Society of London, Series B 281:20141073.

Tabachnick, B. G., and L. S. Fidell. 1989. Using Multivariate Statistics ( $2^{\text {nd }}$ edition). Harper Collins Publishers, New York.

Thomas, M. L., B. Gray, and L. W. Simmons. 2011. Male crickets alter the relative expression of cuticular hydrocarbons when expose to different acoustic environments. Animal Behaviour 82:49-53.

Thomas, M. L., and L. W. Simmons. 2009. Sexual selection on cuticular hydrocarbons in the Australian field cricket, Teleogryllus oceanicus. BMC Evolutionary Biology 9:162.

Thomas, M. L., and L. W. Simmons. 2010. Cuticular hydrocarbons influence female attractiveness to males in the Australian field cricket, Teleogryllus oceanicus. Journal of Evolutionary Biology 23:707-714.

Tinghitella, R. M. 2014. Male and female crickets modulate their courtship behaviour depending on female experience with mate availability. Animal Behaviour

Tinghitella, R. M., and M. Zuk. 2009. Asymmetric mating preferences accommodated the rapid evolutionary loss of a sexual signal. Evolution 63:2087-2098. 
Tinghitella, R. M., M. Zuk, M. Beveridge, and L. W. Simmons. 2011. Island hopping introduces Polynesian field crickets to novel environments, genetic bottlenecks and rapid evolution. Journal of Evolutionary Biology 24:1199-1211.

Tregenza, T., and N. Wedell. 1997. Definitive evidence for cuticular pheromones in a cricket. Animal Behaviour 54:979-984.

Veltsos, P., C. Wicker-Thomas, R. K. Butlin, A. Hoikkala, M. G. Ritchie. 2012. Sexual selection on song and cuticular hydrocarbons in two distinct populations of Drosophila montana. Ecology and Evolution 2:80-94.

Verity, R., and R. A. Nichols. 2014. What is genetic differentiation, and how should we measure it $-G_{S T}, D$, neither, or both? Molecular Ecology 23:4216-4225.

Verzijden, M. N., C. ten Cate, M. R. Servedio, G. M. Kozak, J. W. Boughman, and E. I. Svensson. 2012. The impact of learning on sexual selection and speciation. Trends in Ecology and Evolution 27:511-519.

West-Eberhard, M. J. 1989. Phenotypic plasticity and the origins of diversity. Annual Reviews in Ecology and Systematics 20:249-278.

Whitlock, M. C. 1992. Temporal fluctuations in demographic parameters and the genetic variance among populations. Evolution 46:608-615. 
Wolf, J. B., N. J. Royle, and J. Hunt. 2014. Genotype-by-environment interactions when the social environment contains genes. in Genotype-by-Environment Interactions and Sexual Selection. Eds. J Hunt, and D. J. Hosken. Wiley-Blackwell. 
Table 1. Genetic differentiation in pairwise comparisons between populations. Estimates of $F_{S T}, F_{S T}^{\prime}, G_{S T}^{\prime}(\mathrm{Nei})$, and $D_{E S T}$ (Jost's D) are given in each cell in descending order. P-values for pairwise $F_{S T}$ estimates across all loci are indicated below the diagonal $(P \leq 0.001$ is indicated by asterisks $* * *)$.

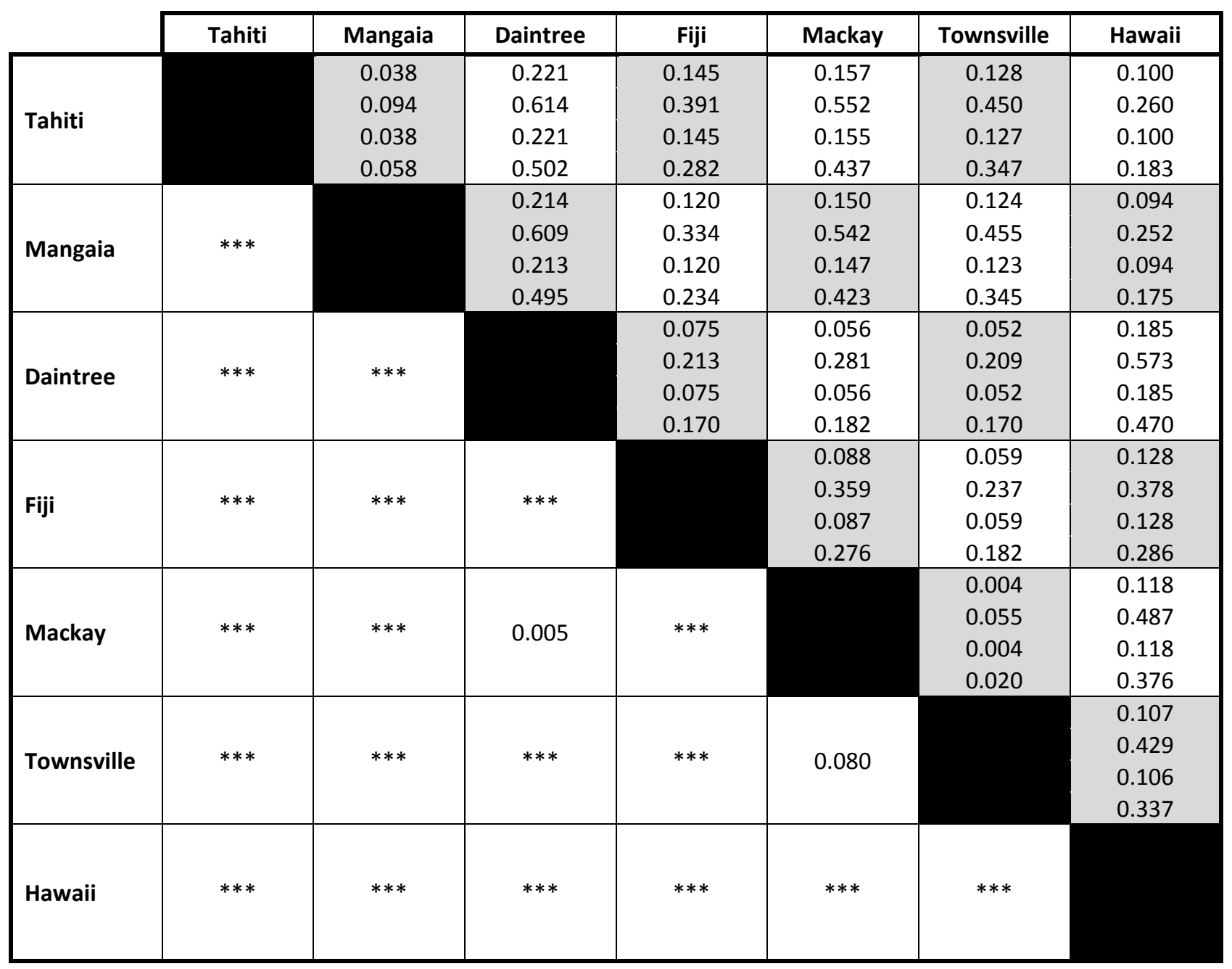


Table 2. Univariate linear models for the PCs describing variation in male $\mathrm{CHC}$ profiles (model 1 ) showing estimated fixed effects and significance assessed from conditional $F$ tests. Note that the "Social" coefficient denotes the effect of experiencing the song treatment relative to no song, while the two "Social.Incubator" treatments denote the difference in trait mean between incubators within the song and no song treatments. Coefficients for "Population" and "Social.Population" are not presented in full here but these effects are shown in Figure 2.

\begin{tabular}{|c|c|c|c|c|c|}
\hline & Model term & Coefficient (SE) & $d f$ & $\boldsymbol{F}$ & $P$ \\
\hline \multirow[t]{7}{*}{ PC1 } & $\mu$ & $-0.087(0.133)$ & 1,751 & 0.00 & 0.996 \\
\hline & PW & $1.142(0.128)$ & 1,751 & 79.49 & $<0.001$ \\
\hline & Social & $0.163(0.187)$ & 1,751 & 14.57 & $<0.001$ \\
\hline & Social.Incubator & $0.014(0.087)$ & 2,751 & 0.03 & 0.971 \\
\hline & & $-0.015(0.084)$ & & & \\
\hline & Population & & 6,751 & 18.95 & $<0.001$ \\
\hline & Social.Population & & 6,751 & 1.34 & 0.237 \\
\hline \multirow[t]{7}{*}{ PC2 } & $\mu$ & $-0.874(0.141)$ & 1,751 & 0.00 & 1.000 \\
\hline & PW & $1.048(0.136)$ & 1,751 & 59.69 & $<0.001$ \\
\hline & Social & $0.102(0.198)$ & 1,751 & 0.52 & 0.470 \\
\hline & Social.Incubator & $0.055(0.092)$ & 2,751 & 0.24 & 0.788 \\
\hline & & $-0.031(0.089)$ & & & \\
\hline & Population & & 6,751 & 36.29 & $<0.001$ \\
\hline & Social.Population & & 6,751 & 0.78 & 0.590 \\
\hline \multirow[t]{7}{*}{ PC3 } & $\mu$ & $0.833(0.127)$ & 1,751 & 0.00 & 1.000 \\
\hline & PW & $-0.003(0.123)$ & 1,751 & 0.00 & 0.980 \\
\hline & Social & $-0.450(0.179)$ & 1,751 & 27.63 & $<0.001$ \\
\hline & Social.Incubator & $-0.049(0.083)$ & 2,751 & 0.71 & 0.495 \\
\hline & & $-0.083(0.081)$ & & & \\
\hline & Population & & 6,751 & 49.35 & $<0.001$ \\
\hline & Social.Population & & 6,751 & 0.86 & 0.521 \\
\hline \multirow[t]{7}{*}{ PC4 } & $\mu$ & $0.182(0.148)$ & 1,751 & 0.00 & 1.000 \\
\hline & PW & $-0.224(0.143)$ & 1,751 & 2.44 & 0.121 \\
\hline & Social & $0.39(0.208)$ & 1,751 & 3.68 & 0.057 \\
\hline & Social.Incubator & $0.087(0.097)$ & 2,751 & 0.75 & 0.472 \\
\hline & & $0.078(0.094)$ & & & \\
\hline & Population & & 6,751 & 17.36 & $<0.001$ \\
\hline & Social.Population & & 6,751 & 1.81 & 0.094 \\
\hline \multirow[t]{6}{*}{ PC5 } & $\mu$ & $-0.246(0.152)$ & 1,751 & 0.00 & 0.991 \\
\hline & PW & $0.092(0.146)$ & 1,751 & 0.39 & 0.528 \\
\hline & Social & $-0.289(0.213)$ & 1,751 & 0.79 & 0.375 \\
\hline & Social.Incubator & $0.042(0.099)$ & 2,751 & 0.38 & 0.681 \\
\hline & & $-0.074(0.096)$ & & & \\
\hline & Population & & 6,751 & 10.43 & $<0.001$ \\
\hline
\end{tabular}

Pascoal et al. Divergence and Social Flexibility page 45 of 65 


\begin{tabular}{|llcccc|} 
& Social.Population & & 6,751 & 1.24 & 0.284 \\
\hline PC6 & $\mu$ & $0.007(0.152)$ & 1,751 & 0.00 & 1.000 \\
& PW & $0.299(0.146)$ & 1,751 & 4.17 & 0.043 \\
& Social & $-0.338(0.214)$ & 1,751 & 0.01 & 0.899 \\
& Social.Incubator & $0.12(0.099)$ & 2,751 & 0.73 & 0.481 \\
& & $-0.009(0.096)$ & & & \\
& & 6,751 & 13.15 & $<0.001$ \\
& Population & & 6,751 & 1.54 & 0.164 \\
\hline
\end{tabular}


Table 3. Estimated genetic (co)variance structure showing among-population variances (diagonal), covariances (below diagonal) and correlations (above diagonal) among CHC traits. Note that phenotypic variances are standardised to 1 so values on diagonal represent the proportion of total variance explained by genetic differentiation. Standard errors are provided in parentheses for all parameter estimates.

\begin{tabular}{|c|c|c|c|c|c|c|}
\cline { 2 - 7 } \multicolumn{1}{c|}{} & PC1 & PC2 & PC3 & PC4 & PC5 & PC6 \\
\hline & 0.127 & -0.793 & 0.369 & 0.237 & -0.415 & -0.577 \\
PC1 & $(0.078)$ & $(0.172)$ & $(0.37)$ & $(0.413)$ & $(0.381)$ & $(0.309)$ \\
\hline & -0.162 & 0.327 & -0.55 & -0.446 & 0.642 & 0.504 \\
PC2 & $(0.109)$ & $(0.194)$ & $(0.294)$ & $(0.347)$ & $(0.273)$ & $(0.332)$ \\
\hline & 0.083 & -0.199 & 0.399 & 0.906 & -0.87 & -0.563 \\
PC3 & $(0.101)$ & $(0.171)$ & $(0.235)$ & $(0.099)$ & $(0.143)$ & $(0.306)$ \\
\hline & 0.035 & -0.107 & 0.241 & 0.177 & -0.900 & -0.579 \\
PC4 & $(0.067)$ & $(0.112)$ & $(0.150)$ & $(0.111)$ & $(0.239)$ & $(0.328)$ \\
\hline & -0.052 & 0.128 & -0.192 & -0.133 & 0.123 & 0.367 \\
PC5 & $(0.059)$ & $(0.102)$ & $(0.124)$ & $(0.085)$ & $(0.083)$ & $(0.399)$ \\
\hline & -0.067 & 0.094 & -0.115 & -0.079 & 0.042 & 0.105 \\
PC6 & $(0.058)$ & $(0.089)$ & $(0.101)$ & $(0.069)$ & $(0.056)$ & $(0.067)$ \\
\hline
\end{tabular}


Table 4. Estimated proportion of among-population variance attributable to drift under model 4 for the six PCs that describe the variation in male $\mathrm{CHC}$ expression. Also shown are LRT comparisons of model 4 to a reduced (drift only) model of among-population differentiation.

\begin{tabular}{|lccccc|}
\hline & $\begin{array}{c}\text { Proportion of genetic } \\
\text { variance explained by } \\
\text { drift (SE) }\end{array}$ & LnL (model 4) & $\operatorname{LnL}$ (drift only) & $\chi^{2}{ }_{1}$ & $P$ \\
\hline PC1 & $0.667(0.571)$ & -262.650 & -261.717 & 1.866 & 0.172 \\
PC2 & $1(-)$ & -305.491 & -305.491 & 0 & 1 \\
\hline PC3 & $1(-)$ & -229.520 & -229.520 & 0 & 1 \\
\hline PC4 & $1(-)$ & -347.499 & -347.499 & 0 & 1 \\
\hline PC5 & $0.951(0.171)$ & -361.356 & -361.263 & 0.186 & 0.666 \\
\hline PC6 & $1(-)$ & -363.243 & -363.243 & 0 & 1 \\
\hline
\end{tabular}




\section{FIGURE LEGENDS}

Figure 1. Teleogryllus oceanicus population genetics. (A) Stars indicate populations studied. The arrows show putative eastward range expansion (Tinghitella et al. 2011). (B) Unrooted neighbour-joining tree based on microsatellite allele frequencies, constructed using Nei's (1983) genetic distance, Da. (C) Allelic diversity in each population. Circles indicate means and error bars represent one standard error.

Figure 2. The separation of male $\mathrm{CHC}$ expression across populations according to the first two discriminant functions (DF1 and DF2, CHC peak loadings are provided in Table S5 in the Supporting Information). Together, these functions explain $78.9 \%$ of the variation in male $\mathrm{CHC}$ expression. Individual data points are provided as circles and the population centroids in squares (with crosshairs). Populations are colour-coded to match Figure 1.

Figure 3. Reaction norms showing the response of the six PCS $(A-F)$ describing variation in male CHCs in each population to the social environment. Mean PC values in each population and environment are expressed as Best Linear Unbiased Estimators (BLUEs) from a linear model. Populations are colour-coded to match Figure 1. 

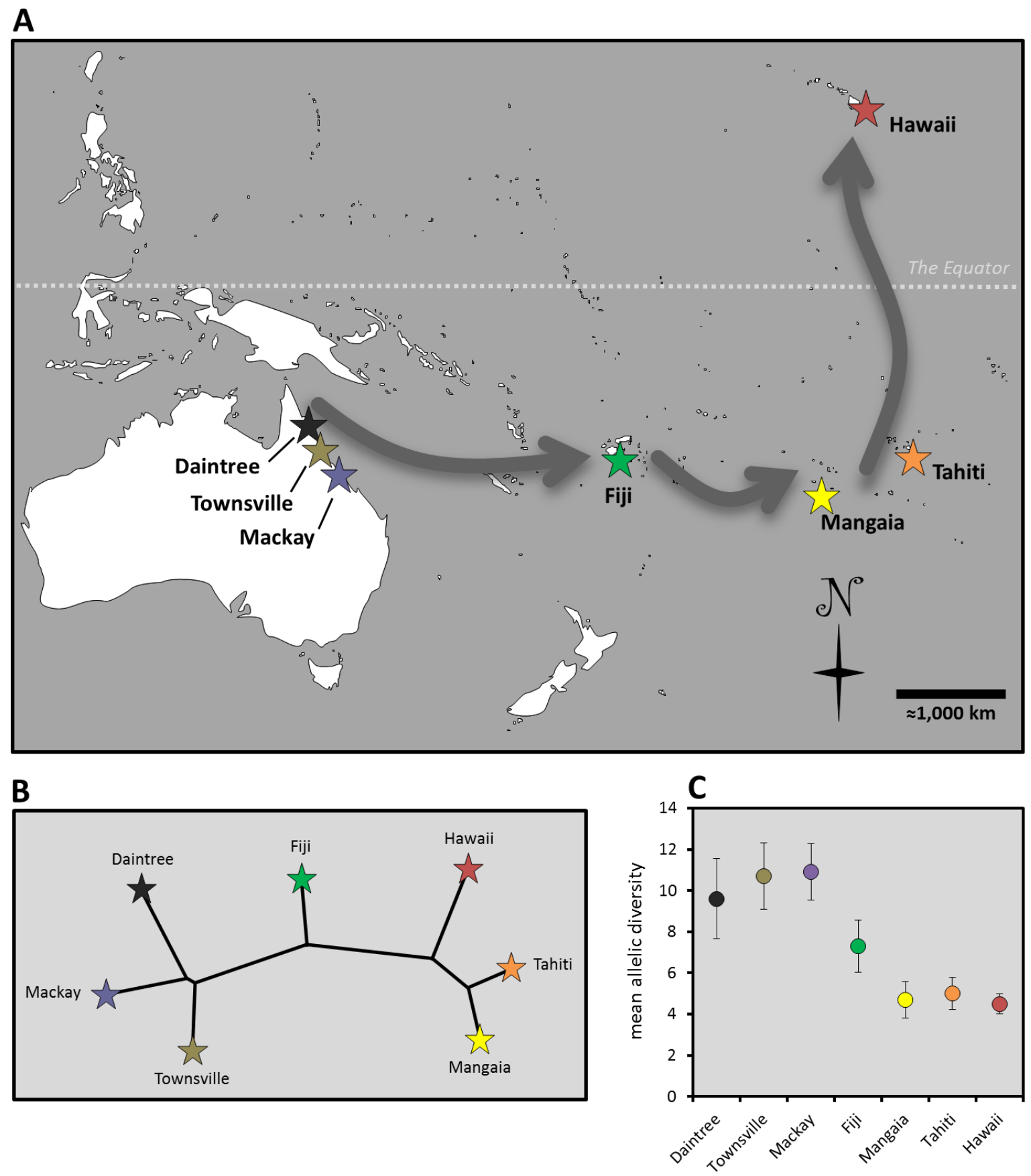

Figure 1. 




Figure 2. 



Social Environment

Figure 3. 


\section{Online Supporting Information For:}

\section{Sexual Selection and Population Divergence I. the InfLUENCE OF SOCIALLY FLEXIBLE CUTICULAR HydROCARBON EXPRESSION IN MALE FIELD CRICKETS (TELEOGRYLLUS OCEANICUS)}

Sonia Pascoal ${ }^{1}$, Magdalena Mendrok ${ }^{2}$, Christopher Mitchell ${ }^{3,4}$, Alastair J. Wilson ${ }^{4}$, John Hunt ${ }^{4}$, Nathan W. Bailey ${ }^{1,5}$

${ }^{1}$ Centre for Biological Diversity, University of St Andrews, St Andrews, KY16 9TH, United Kingdom

${ }^{2}$ Institute of Environmental Sciences, Jagellonian University, Gronostajova 7, 30-387 Kraków, Poland

${ }^{3}$ Hawkesbury Institute for the Environment, University of Western Sydney, Penrith NSW 2751, Australia

${ }^{4}$ Centre for Ecology and Conservation, College of Life and Environmental Sciences, University of Exeter, Cornwall Campus, TR10 9EZ, United Kingdom

${ }^{5}$ E-mail: nwb3@st-andrews.ac.uk 


\section{Contents}

p. 3 ......... Figure S1. Example chromatograph for male T. oceanicus $\mathrm{CHCs}$.

p. 4 ......... Table S1. Details of $T$. oceanicus population origins and sample sizes.

p. 5 ......... Table S2. Microsatellite primer sequences, PCR conditions, and allele information.

p. 6 .......... Table S3. Male calling song data and playback song details.

p. 7 ......... Table S4. Identification of cuticular hydrocarbon compounds in male $T$. oceanicus.

p. 8 ......... Table S5. Principal component analysis of male cuticular hydrocarbons (CHCs).

p. 9 ......... Table S6. Univariate mixed models of $\mathrm{CHC}$ expression profiles.

p. 10 ....... Table S7. Post-hoc analysis of socially flexible CHC expression examining differences between mainland Australian and Oceanic island populations.

p. 12 ....... Table S8. Discriminant function analysis examining population differences in $\mathrm{CHC}$ profiles. 


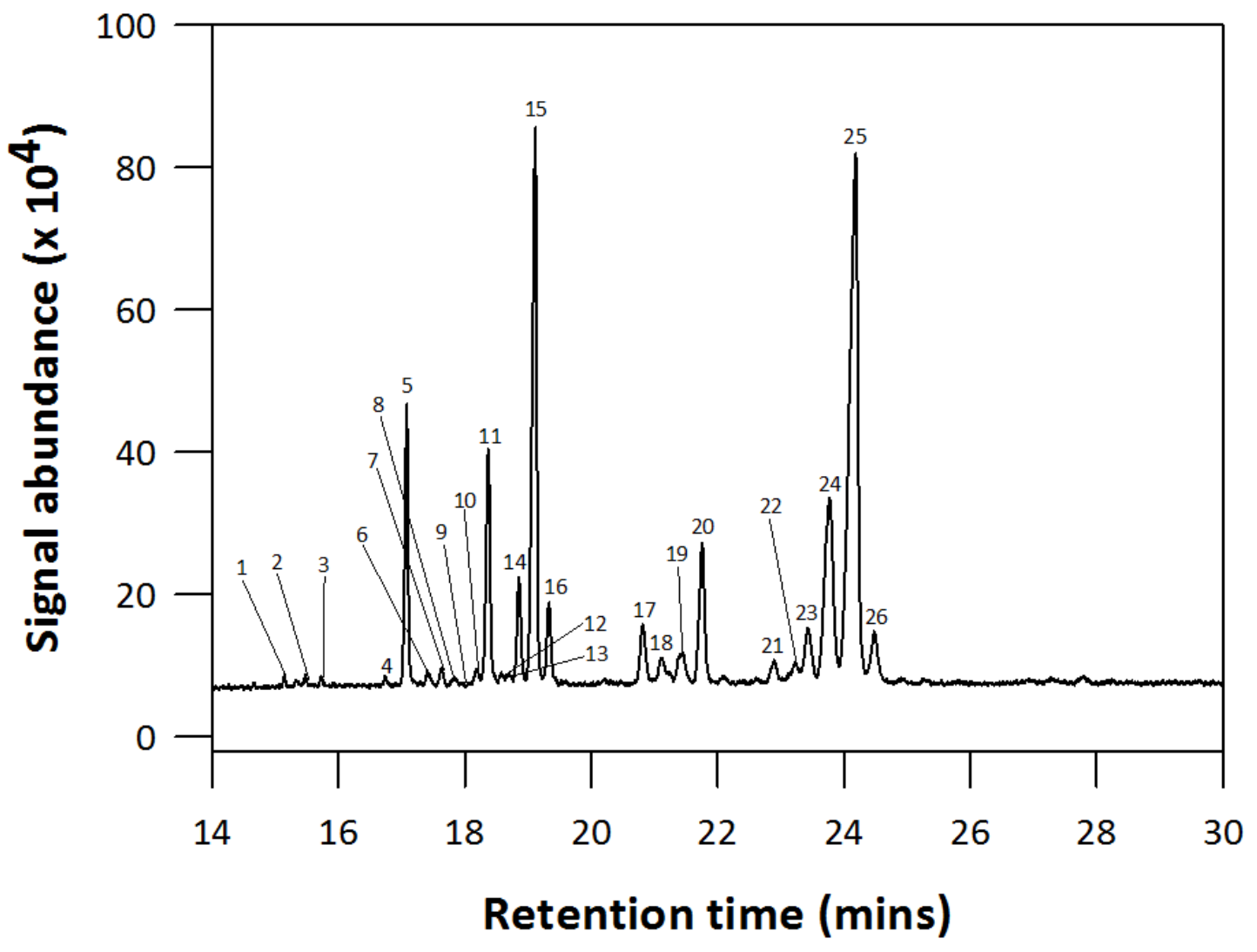

Figure S1. A chromatograph of a typical cuticular hydrocarbon $(\mathrm{CHC})$ profile of a male Teleogryllus oceanicus. Peak 1 represents the internal standard (pentadecane) and the remaining 26 peaks represent the individual CHCs identified in Table S3. 
Table S1. Details of $T$. oceanicus population origins and sample sizes.

\begin{tabular}{|c|c|c|c|c|}
\hline \multirow{3}{*}{$\begin{array}{l}\text { population } \\
\text { location }\end{array}$} & \multirow{3}{*}{$\begin{array}{l}\text { year } \\
\text { established }\end{array}$} & microsatellite & $\begin{array}{c}\text { male calling } \\
\text { song }\end{array}$ & $\begin{array}{c}\mathrm{CHC} \\
\text { extraction }\end{array}$ \\
\hline & & $\boldsymbol{N}$ females & $N$ females & $N$ females \\
\hline & & $N$ males & $N$ males & $N$ males \\
\hline \multirow{2}{*}{$\begin{array}{l}\text { Daintree, } \\
\text { Australia }\end{array}$} & \multirow{2}{*}{2011} & 9 & - & - \\
\hline & & 15 & 19 & 97 \\
\hline \multirow{2}{*}{$\begin{array}{l}\text { Townsville, } \\
\text { Australia }\end{array}$} & \multirow{2}{*}{2011} & 15 & - & - \\
\hline & & 9 & 19 & 122 \\
\hline \multirow{2}{*}{$\begin{array}{l}\text { Mackay, } \\
\text { Australia }\end{array}$} & \multirow{2}{*}{2011} & 22 & - & - \\
\hline & & 2 & 20 & 89 \\
\hline \multirow{2}{*}{ Fiji } & \multirow{2}{*}{2010} & 8 & - & - \\
\hline & & 16 & 19 & 115 \\
\hline \multirow{2}{*}{ Mangaia } & \multirow{2}{*}{2009} & 8 & - & - \\
\hline & & 16 & 21 & 109 \\
\hline \multirow{2}{*}{ Tahiti } & \multirow{2}{*}{2009} & 8 & - & - \\
\hline & & 16 & 21 & 112 \\
\hline \multirow{2}{*}{ Hawaii } & \multirow{2}{*}{$2012^{+}$} & 12 & - & - \\
\hline & & 12 & 18 & 124 \\
\hline
\end{tabular}

${ }^{\dagger}$ eggs collected on approximately yearly field trips, with the last collection occurring on the indicated date.

*DNA extracted from wild-caught individuals. 
Table S2. Primer sequences and PCR reaction conditions for microsatellite loci. Summary population genetic statistics are indicated for each locus; these were estimated using FSTAT v.1.2 (Goudet 1995) as described in the main text.

\begin{tabular}{|c|c|c|c|c|c|c|c|}
\hline Locus & Primer sequence 5'-3' and labelling & Repeat motif & Size range (bp) & $\mathbf{T}_{\mathrm{A}}$ & $\mathbf{N}_{\mathrm{A}}$ & $\mathbf{H}_{\mathrm{E}}$ & $\mathbf{H}_{\mathbf{O}}$ \\
\hline \multirow{2}{*}{ contig07712* } & F: FAM-CCTGTTGTATCCAGTGCACG & \multirow{2}{*}{$(\mathrm{AG})_{8}$} & \multirow{2}{*}{$421-433$} & \multirow{2}{*}{55} & \multirow{2}{*}{7} & \multirow{2}{*}{0.548} & \multirow{2}{*}{0.502} \\
\hline & R: TGTGCACACTATACTTCAGCAC & & & & & & \\
\hline \multirow{2}{*}{ contig12396* } & F: PET-AGCACTTCTCTCTCATGGCC & \multirow{2}{*}{$(\mathrm{AT})_{8}$} & \multirow{2}{*}{$286-306$} & \multirow{2}{*}{55} & \multirow{2}{*}{8} & \multirow{2}{*}{0.602} & \multirow{2}{*}{0.813} \\
\hline & R: TGAATGAGCGAATGTGAAGAGC & & & & & & \\
\hline \multirow{2}{*}{ contig27208* } & R: FAM-CCTTAAGGTCTGAGCGTCCC & \multirow{2}{*}{$(\mathrm{AAC})_{12}$} & \multirow{2}{*}{$249-312$} & \multirow{2}{*}{55} & \multirow{2}{*}{18} & \multirow{2}{*}{0.695} & \multirow{2}{*}{0.788} \\
\hline & F: TACCGCACTGTATCGCAATC & & & & & & \\
\hline \multirow{2}{*}{ contig39588* } & F: FAM-GTGAATGCGTGCGAGTGG & \multirow{2}{*}{$(\mathrm{AC})_{8}$} & \multirow{2}{*}{$96-134$} & \multirow{2}{*}{55} & \multirow{2}{*}{14} & \multirow{2}{*}{0.639} & \multirow{2}{*}{0.783} \\
\hline & R: TGGACCACGGGACATGATTG & & & & & & \\
\hline \multirow{2}{*}{ Totri9a } & F: NED-CCATTCCCTGATCCTGAGAGT & \multirow{2}{*}{$(\mathrm{GAT})_{5}$} & \multirow{2}{*}{$127-160$} & \multirow{2}{*}{55} & \multirow{2}{*}{10} & \multirow{2}{*}{0.688} & \multirow{2}{*}{0.690} \\
\hline & R: GACTGAGCCGAGCAATTCAA & & & & & & \\
\hline & F: FAM-TGACCTGTGGTTGCTTCTAGC & & & & & & \\
\hline Totri54 & R: CTGAACCAGCACAAACAGGA & $(\text { CAT })_{8}$ & $90-147$ & 55 & 17 & 0.799 & 0.945 \\
\hline & F: VIC-AGGTCAGGCGGATACAAACT & & & & & & \\
\hline Totris5a & R CTGCGAATGACACATCATTG & $(G A A)_{16}$ & $81-225$ & 55 & 34 & 0.893 & 0.988 \\
\hline Totri57 & F: PET-ACAGACAGCAATGGGTGACA & (GAT) & 181,2014 & 55 & 16 & 0602 & 0709 \\
\hline (1007) & R: TACCCTCGAACGCTTCGTC & $(\mathrm{GAT} / 12$ & $101-\angle 44$ & ככ & 10 & 0.092 & 0.194 \\
\hline Totri78 & F: VIC-ATAGGCTCAACGCCAGCA & 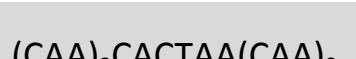 & 127102 & 55 & 11 & 0712 & 0761 \\
\hline $10011 / 8$ & F: GTGCCAGTATCTGATATTTTGTGC & A/9 9 & $13<-183$ & 35 & 14 & 0.143 & 0.101 \\
\hline Totri88a & R: NED-AGGACCGGATTGGAGAGTAA & $(G A A) G A G(G A A)$ & $138-174$ & 55 & 10 & 0617 & 0636 \\
\hline & F: TCACCGCGTCTCTCTTTTCA & & & & & & \\
\hline
\end{tabular}

${ }^{*}$ microsatellite loci developed in the present study, $\mathrm{T}_{\mathrm{A}}$ : annealing temperature $\left({ }^{\circ} \mathrm{C}\right), \mathrm{N}_{\mathrm{A}}$ : number of alleles per locus, $\mathrm{H}_{\mathrm{E}}$ : expected heterozygosity, $\mathrm{H}_{\mathrm{O}}$ : observed heterozygosity. 
Table S3. Average calling song parameters for (A) each T. oceanicus population, (B) combined across all populations, (C) each song playback. Song measurements are in seconds unless otherwise noted and are based on raw data. Data from Townsville and Daintree have been previously published (Bailey and Macleod 2014). Standard errors were calculated after averaging all 10 songs per individual.

\begin{tabular}{|c|c|c|c|c|c|c|c|c|c|c|c|c|c|c|c|c|c|c|c|c|c|c|c|}
\hline & \multicolumn{14}{|c|}{$\begin{array}{c}\text { (A) } \\
\text { POPULATION MEANS }\end{array}$} & \multirow{2}{*}{\multicolumn{2}{|c|}{$\begin{array}{c}\text { (B) } \\
\text { COMBINED } \\
(n=137)\end{array}$}} & \multicolumn{7}{|c|}{$\begin{array}{c}\text { (C) } \\
\text { PLAYBACK }\end{array}$} \\
\hline & \multicolumn{2}{|c|}{$\begin{array}{l}\text { Daintree } \\
(n=19)\end{array}$} & \multicolumn{2}{|c|}{$\begin{array}{c}\text { Townsville } \\
(n=19)\end{array}$} & \multicolumn{2}{|c|}{$\begin{array}{l}\text { Mackay } \\
(n=20)\end{array}$} & \multicolumn{2}{|c|}{$\begin{array}{c}\text { Fiji } \\
(n=19)\end{array}$} & \multicolumn{2}{|c|}{$\begin{array}{l}\text { Mangaia } \\
(n=21)\end{array}$} & \multicolumn{2}{|c|}{$\begin{array}{l}\text { Tahiti } \\
(n=21)\end{array}$} & \multicolumn{2}{|c|}{$\begin{array}{l}\text { Hawaii } \\
(n=18)\end{array}$} & & & & & & & & & \\
\hline & $\bar{x}$ & s.e. & $\bar{x}$ & s.e. & $\bar{x}$ & s.e. & $\bar{x}$ & s.e. & $\bar{x}$ & s.e. & $\bar{x}$ & s.e. & $\bar{x}$ & s.e. & $\bar{x}$ & s.e. & 1 & 2 & 3 & 4 & 5 & 6 & $\bar{x}$ \\
\hline total song length & 1.594 & 0.0653 & 1.581 & 0.0702 & 1.686 & 0.0789 & 1.322 & 0.0666 & 1.164 & 0.0502 & 1.364 & 0.0815 & 1.277 & 0.0510 & 1.425 & 0.0294 & 1.086 & 1.900 & 0.989 & 1.503 & 1.169 & 1.516 & 1.361 \\
\hline long chirp length & 0.307 & 0.0139 & 0.305 & 0.0131 & 0.285 & 0.0090 & 0.295 & 0.0091 & 0.381 & 0.0105 & 0.378 & 0.0129 & 0.383 & 0.0236 & 0.334 & 0.0062 & 0.358 & 0.292 & 0.290 & 0.357 & 0.293 & 0.294 & 0.314 \\
\hline $\begin{array}{l}\text { number of long chirp } \\
\text { pulses }\end{array}$ & 5.41 & 0.2690 & 5.32 & 0.2010 & 4.95 & 0.1390 & 5.08 & 0.1440 & 6.06 & 0.1510 & 5.84 & 0.1650 & 6.24 & 0.3300 & 5.56 & 0.0854 & 6.00 & 5.00 & 5.00 & 6.00 & 5.00 & 5.00 & 5.33 \\
\hline $\begin{array}{l}\text { long chirp pulse } \\
\text { length }\end{array}$ & 0.037 & 0.0009 & 0.036 & 0.0007 & 0.037 & 0.0009 & 0.034 & 0.0009 & 0.030 & 0.0011 & 0.036 & 0.0012 & 0.037 & 0.0009 & 0.035 & 0.0004 & 0.036 & 0.035 & 0.035 & 0.035 & 0.035 & 0.035 & 0.035 \\
\hline $\begin{array}{l}\text { long chirp inter-pulse } \\
\text { interval }\end{array}$ & 0.024 & 0.0008 & 0.026 & 0.0007 & 0.026 & 0.0010 & 0.027 & 0.0012 & 0.038 & 0.0012 & 0.031 & 0.0012 & 0.027 & 0.0010 & 0.029 & 0.0005 & 0.029 & $\begin{array}{l}0.028 \\
\end{array}$ & 0.028 & $\begin{array}{l}0.029 \\
\end{array}$ & \begin{tabular}{l|l}
0.029 \\
\end{tabular} & 0.029 & 0.029 \\
\hline $\begin{array}{l}\text { long chirp - short } \\
\text { chirp interval }\end{array}$ & 0.067 & 0.0021 & 0.071 & 0.0038 & 0.072 & 0.0026 & 0.053 & 0.0020 & 0.072 & 0.0023 & 0.072 & 0.0036 & 0.064 & 0.0019 & 0.068 & 0.0011 & 0.070 & 0.055 & 0.057 & 0.048 & 0.059 & 0.112 & 0.067 \\
\hline $\begin{array}{l}\text { total short chirp } \\
\text { length }\end{array}$ & 1.227 & 0.0562 & 1.206 & 0.0625 & 1.334 & 0.0774 & 0.975 & 0.0621 & 0.710 & 0.0442 & 0.917 & 0.0732 & 0.828 & 0.0494 & 1.026 & 0.0295 & 0.658 & 1.551 & 0.640 & 1.098 & 0.815 & 1.109 & 0.979 \\
\hline $\begin{array}{l}\text { number of short } \\
\text { chirps }\end{array}$ & 8.21 & 0.31 & 8.10 & 0.37 & 8.84 & 0.45 & 7.15 & 0.33 & 4.90 & 0.25 & 5.90 & 0.38 & 5.64 & 0.29 & 6.94 & 0.1755 & 5.00 & 11.00 & 5.00 & 8.00 & 6.00 & 8.00 & 7.17 \\
\hline paired pulse length & 0.069 & 0.0011 & 0.067 & 0.0010 & 0.068 & 0.0011 & 0.066 & 0.0012 & 0.069 & 0.0014 & 0.072 & 0.0015 & 0.078 & 0.0018 & 0.070 & 0.0006 & 0.067 & 0.068 & 0.064 & 0.067 & 0.068 & 0.068 & 0.067 \\
\hline $\begin{array}{l}\text { short chirp inter- } \\
\text { chirp interval }\end{array}$ & 0.076 & 0.0040 & 0.080 & 0.0039 & 0.085 & 0.0041 & 0.068 & 0.0033 & 0.088 & 0.0026 & 0.088 & 0.0040 & 0.077 & 0.0027 & 0.081 & 0.0014 & 0.080 & 0.079 & 0.080 & 0.080 & 0.080 & 0.080 & 0.080 \\
\hline $\begin{array}{l}\text { short chirp pulse } \\
\text { length }\end{array}$ & 0.029 & 0.0006 & 0.027 & 0.0006 & 0.028 & 0.0006 & 0.028 & 0.0007 & 0.027 & 0.0009 & 0.029 & 0.0009 & 0.031 & 0.0008 & 0.028 & 0.0003 & 0.026 & 0.027 & 0.025 & 0.026 & 0.027 & 0.027 & 0.026 \\
\hline $\begin{array}{l}\text { short chirp inter- } \\
\text { pulse interval }\end{array}$ & 0.012 & 0.0003 & 0.012 & 0.0004 & 0.013 & 0.0005 & 0.011 & 0.0005 & 0.017 & 0.0007 & 0.014 & 0.0005 & 0.014 & 0.0006 & 0.013 & 0.0003 & 0.013 & 0.013 & 0.013 & 0.013 & \begin{tabular}{|l|l}
0.014 \\
\end{tabular} & 0.013 & 0.013 \\
\hline inter-song interval & 0.099 & 0.0055 & 0.105 & 0.0075 & 0.106 & 0.0052 & 0.081 & 0.0046 & 0.109 & 0.0076 & 0.122 & 0.0076 & 0.097 & 0.0078 & 0.103 & 0.0027 & 0.109 & 0.182 & 0.046 & 0.089 & 0.046 & 0.114 & 0.098 \\
\hline $\begin{array}{l}\text { mean pulses per } \\
\text { short chirp }\end{array}$ & 2.28 & 0.0620 & 2.26 & 0.0560 & 2.12 & 0.0546 & 2.20 & 0.0492 & 2.08 & 0.0256 & 2.05 & 0.0154 & 2.05 & 0.0163 & 2.16 & 0.0178 & 2.00 & 2.00 & 2.00 & 2.00 & 2.00 & 2.00 & 2.00 \\
\hline $\begin{array}{l}\text { carrier frequency } \\
(\mathrm{Hz})\end{array}$ & 5087 & 40.4 & 5029 & 64.0 & 5038 & 32.4 & 5070 & 50.3 & 5126 & 46.5 & 4897 & 32.5 & 4926 & 42.9 & 5025 & 17.9 & 5070 & 4599 & 5429 & 5028 & 4899 & 4966 & 4999 \\
\hline
\end{tabular}


Table S4. Identification of the 26 cuticular hydrocarbon compounds in male Teleogryllus oceanicus (see Figure S1). The peak numbers correspond with those presented on the chromatograph (Figure S1). KRI is the Kováts retention index calculated according to Majlát et al. (1974) in comparison to $n$-alkane standards $\left(C_{7}-C_{40}\right)$. The diagnostic ions presented in bold were used during identification with dimethyl-disulphide (DMDS) derivation.

\begin{tabular}{|c|c|c|c|}
\hline Peak number & KRI & ID & Diagnostic ions \\
\hline Std & & Pentadecane & \\
\hline 1 & 2840 & $4 \mathrm{MeC}_{28}$ & 365,71 \\
\hline 2 & 2893 & $10 \mathrm{MeC}_{28}$ & 281,155 \\
\hline 3 & 2910 & $13 \mathrm{MeC}_{29}$ & 252,196 \\
\hline 4 & 3028 & $C_{30: 1}$ & 434 \\
\hline 5 & 3043 & $4 \mathrm{MeC}_{30}$ & $436,393,71$ \\
\hline 6 & 3064 & 7,x-diMeC 30 & 365,112 \\
\hline 7 & 3075 & Unidentified & \\
\hline 8 & 3086 & $C_{31: 1}$ & 434 \\
\hline 9 & 3110 & $11 \mathrm{MeC}_{31}$ & 308,168 \\
\hline 10 & 3119 & $7,8 \mathrm{MeC}_{31}$ & 364,112 \\
\hline 11 & 3130 & 7- $C_{31}$ ene & $434,528,145,383$ (DMDS) \\
\hline 12 & 3142 & $C_{31: 1}$ & 434 \\
\hline 13 & 3152 & $C_{31: 2}$ & 432 \\
\hline 14 & 3161 & $C_{31: 2}$ & 432 \\
\hline 15 & 3174 & $C_{31: 2}$ & 432 \\
\hline 16 & 3188 & $C_{31: 2}$ & 432 \\
\hline 17 & 3242 & $4 \mathrm{MeC}_{32}$ & 421,71 \\
\hline 18 & 3252 & Unidentified & \\
\hline 19 & 3268 & $C_{32: 2}$ & 446 \\
\hline 20 & 3288 & $C_{33: 1}$ & 462 \\
\hline 21 & 3331 & $C_{33: 1}$ & 462 \\
\hline 22 & 3347 & $C_{33: 2}$ & 460 \\
\hline 23 & 3355 & $C_{33: 2}$ & 460 \\
\hline 24 & 3365 & $C_{33: 2}$ & 460 \\
\hline 25 & 3379 & $3, x-C_{33: 2}$ & $460,647,89$ (DMDS) \\
\hline 26 & 3391 & $\mathrm{C}_{33: 2}$ & 460 \\
\hline
\end{tabular}


Table S5. Principal Component (PC) analysis of male cuticular hydrocarbons (CHCs) in Teleogryllus oceanicus. Peak numbers refer to those identified in Table S3 and Figure S1, with the exception that "Std" is not included as this is the internal standard (pentadecane). We retained PCs with an eigenvalue greater than 1 in our subsequent analyses and we interpret factor loading $>|0.30|$ as biologically significant (in bold) (Tabachnick and Fidell 1989).

\begin{tabular}{|c|c|c|c|c|c|c|}
\hline & PC1 & PC2 & PC3 & PC4 & PC5 & PC6 \\
\hline Eigenvalue & 9.985 & 4.299 & 1.894 & 1.336 & 1.187 & 1.020 \\
\hline Variance explained (\%) & 38.402 & 16.533 & 7.384 & 5.139 & 4.564 & 3.923 \\
\hline \multicolumn{7}{|l|}{ CHC loadings (peak \#) } \\
\hline $4 \mathrm{MeC}_{28}($ peak 1$)$ & 0.559 & 0.159 & 0.358 & -0.055 & 0.300 & -0.139 \\
\hline $10 \mathrm{MeC}_{28}$ (peak 2) & 0.683 & -0.240 & 0.131 & 0.043 & -0.039 & 0.070 \\
\hline $13 \mathrm{MeC}_{29}$ (peak 3) & 0.765 & -0.194 & -0.089 & -0.220 & 0.263 & -0.004 \\
\hline $\mathrm{C}_{30: 1}($ peak 4) & 0.699 & -0.184 & 0.176 & -0.074 & -0.019 & 0.113 \\
\hline $4 \mathrm{MeC}_{30}($ peak 5) & 0.729 & -0.257 & -0.405 & -0.243 & 0.048 & 0.065 \\
\hline 7,x-diMeC 30 (peak 6) & 0.661 & -0.203 & 0.470 & 0.215 & -0.281 & -0.134 \\
\hline Unidentified (peak 7) & 0.738 & -0.203 & -0.289 & -0.078 & 0.238 & -0.089 \\
\hline$C_{31: 1}($ peak 8) & 0.737 & 0.030 & 0.121 & -0.107 & 0.381 & -0.076 \\
\hline $11 \mathrm{MeC}_{31}$ (peak 9) & 0.227 & -0.017 & 0.075 & -0.007 & 0.329 & 0.631 \\
\hline $7,8 \mathrm{MeC}_{31}($ peak 10) & 0.686 & -0.229 & 0.504 & 0.179 & -0.346 & -0.031 \\
\hline 7- $C_{31}$ ene (peak 11) & 0.801 & -0.331 & -0.108 & -0.124 & -0.268 & 0.250 \\
\hline $\mathrm{C}_{31: 1}$ (peak 12) & 0.672 & 0.074 & 0.389 & -0.302 & -0.040 & 0.067 \\
\hline$C_{31: 2}$ (peak 13) & 0.471 & -0.377 & 0.345 & -0.343 & 0.111 & -0.096 \\
\hline $\mathrm{C}_{31: 2}$ (peak 14) & 0.748 & -0.358 & -0.233 & 0.245 & 0.044 & -0.046 \\
\hline $\mathrm{C}_{31: 2}($ peak 15) & 0.819 & -0.425 & -0.108 & 0.047 & -0.203 & -0.026 \\
\hline $\mathrm{C}_{31: 2}$ (peak 16) & 0.762 & -0.322 & -0.097 & -0.247 & -0.045 & 0.018 \\
\hline $4 \mathrm{MeC}_{32}$ (peak 17) & 0.009 & 0.867 & 0.046 & -0.017 & 0.006 & 0.001 \\
\hline Unidentified (peak 18) & 0.589 & 0.473 & 0.106 & -0.082 & 0.131 & -0.290 \\
\hline$C_{32: 2}$ (peak 19) & 0.634 & 0.574 & 0.035 & 0.147 & 0.018 & -0.248 \\
\hline $\mathrm{C}_{33: 1}($ peak 20) & 0.471 & 0.545 & -0.549 & -0.023 & 0.047 & -0.164 \\
\hline $\mathrm{C}_{33: 1}($ peak 21) & 0.166 & 0.757 & 0.173 & -0.025 & -0.173 & 0.378 \\
\hline $\mathrm{C}_{33: 2}$ (peak 22) & 0.370 & 0.220 & 0.101 & 0.513 & 0.337 & 0.327 \\
\hline $\mathrm{C}_{33: 2}$ (peak 23) & 0.579 & 0.195 & -0.067 & 0.617 & 0.149 & -0.076 \\
\hline $\mathrm{C}_{33: 2}($ peak 24) & 0.759 & 0.314 & -0.253 & 0.232 & -0.258 & -0.020 \\
\hline $3, x-C_{33: 2}($ peak 25) & 0.607 & 0.494 & -0.431 & -0.044 & -0.317 & 0.179 \\
\hline$C_{33: 2}$ (peak 26) & 0.116 & 0.817 & 0.024 & -0.211 & -0.174 & 0.087 \\
\hline
\end{tabular}


Table S6. Discriminant Function (DF) analysis separating the variation in male $\mathrm{CHC}$ expression in Teleogryllus oceanicus according to population of origin. We used Wilks' Lambda $(\lambda)$ values to determine which DFs were statistically significant and interpret factor loadings $>|0.30|$ as biologically significant (in bold) (Tabachnick and Fidell 1989).

\begin{tabular}{|c|c|c|c|c|c|c|}
\hline & DF1 & DF2 & DF3 & DF4 & DF5 & DF6 \\
\hline Eigenvalue & 3.472 & 0.707 & 0.614 & 0.325 & 0.103 & 0.075 \\
\hline Wilks' Lambda $(\lambda)$ & 0.052 & 0.231 & 0.394 & 0.636 & 0.843 & 0.930 \\
\hline$d f$ & 156 & 125 & 96 & 69 & 44 & 21 \\
\hline$P$ & 0.0001 & 0.0001 & 0.0001 & 0.0001 & 0.0001 & 0.0001 \\
\hline$\%$ variance explained & 65.560 & 13.348 & 11.592 & 6.138 & 1.941 & 1.421 \\
\hline \multicolumn{7}{|l|}{ CHC loadings (peak \#) } \\
\hline $4 \mathrm{MeC}_{28}($ peak 1) & 0.158 & -0.418 & 0.350 & 0.506 & 0.053 & -0.067 \\
\hline $10 \mathrm{MeC}_{28}$ (peak 2) & -0.240 & -0.129 & -0.070 & 0.438 & -0.505 & -0.235 \\
\hline $13 \mathrm{MeC}_{29}$ (peak 3) & -0.185 & 0.012 & 0.091 & -0.579 & 0.002 & 0.020 \\
\hline $\mathrm{C}_{30: 1}($ peak 4) & 0.015 & 0.350 & 0.167 & -0.028 & 0.033 & -0.197 \\
\hline $4 \mathrm{MeC}_{30}($ peak 5) & -0.608 & -0.817 & -0.184 & 0.229 & -0.494 & 0.824 \\
\hline 7,x-diMeC 30 (peak 6) & 0.357 & 0.153 & -0.537 & 0.172 & -0.402 & -0.308 \\
\hline Unidentified (peak 7) & -0.124 & -0.018 & -0.456 & 0.149 & 0.427 & 0.232 \\
\hline$C_{31: 1}($ peak 8) & -0.023 & -0.241 & -0.050 & -0.195 & -0.073 & -0.297 \\
\hline $11 \mathrm{MeC}_{31}$ (peak 9) & -0.087 & -0.183 & 0.091 & 0.058 & -0.025 & 0.013 \\
\hline $7,8 \mathrm{MeC}_{31}$ (peak 10) & 0.351 & -0.448 & -0.246 & 0.323 & 0.392 & 0.208 \\
\hline 7- $C_{31}$ ene (peak 11) & 0.648 & 0.217 & 0.493 & -0.062 & 1.441 & -0.545 \\
\hline$C_{31: 1}$ (peak 12) & 0.080 & -0.002 & -0.188 & -0.159 & 0.022 & 0.210 \\
\hline$C_{31: 2}$ (peak 13) & -0.026 & 0.095 & -0.123 & -0.078 & 0.033 & 0.094 \\
\hline$C_{31: 2}$ (peak 14) & -0.190 & 0.378 & -0.105 & 0.007 & 0.117 & 0.083 \\
\hline$C_{31: 2}$ (peak 15) & 0.869 & 0.705 & 1.368 & -0.257 & 0.085 & 0.424 \\
\hline$C_{31: 2}$ (peak 16) & -0.334 & -0.087 & -0.164 & -0.009 & -0.466 & -0.468 \\
\hline $4 \mathrm{MeC}_{32}$ (peak 17) & 0.512 & 1.166 & -0.104 & 0.310 & 0.097 & -0.033 \\
\hline Unidentified (peak 18) & 0.143 & 0.377 & 0.151 & 0.068 & -0.378 & 0.048 \\
\hline$C_{32: 2}$ (peak 19) & 0.167 & -0.654 & 0.137 & -0.240 & 0.175 & 0.466 \\
\hline$C_{33: 1}$ (peak 20) & -0.407 & 0.088 & 0.783 & 1.107 & 1.154 & -0.107 \\
\hline$C_{33: 1}$ (peak 21) & -0.391 & -0.178 & -0.161 & -0.228 & 0.719 & -0.079 \\
\hline $\mathrm{C}_{33: 2}$ (peak 22) & 0.009 & 0.191 & -0.280 & 0.007 & 0.059 & 0.035 \\
\hline $\mathrm{C}_{33: 2}$ (peak 23) & 0.112 & 0.125 & 0.067 & -0.084 & -0.147 & -0.057 \\
\hline$C_{33: 2}$ (peak 24) & 0.578 & 0.107 & -0.163 & -0.707 & -0.465 & 0.609 \\
\hline $3, x-C_{33: 2}$ (peak 25) & -0.871 & -0.495 & -1.043 & -0.543 & -1.102 & -0.926 \\
\hline $\mathrm{C}_{33: 2}$ (peak 26) & -0.038 & 0.017 & 0.302 & 0.089 & 0.025 & 0.727 \\
\hline
\end{tabular}


Table S7. Post-hoc analysis of socially flexible CHC expression examining differences between mainland Australian and Oceanic island populations ("Landtype"). The six principal components describing male $\mathrm{CHC}$ variation are modelled separately. Arrows in the left column indicate the interaction term which, if significant, would indicate that mainland and island populations respond differently to acoustic experience. Unlike the models above, population-level variation is controlled by nesting Population within Landtype. We also included a nesting term to test whether populations within a given landtype showed variable social flexibility.

\begin{tabular}{|c|c|c|c|c|}
\hline & Model term & $d f$ & $\boldsymbol{F}$ & $\boldsymbol{P}$ \\
\hline \multirow[t]{10}{*}{ PC1 } & $\mu$ & 1,751 & 0.10 & 0.748 \\
\hline & PW & 1,751 & 79.49 & $<0.001$ \\
\hline & Social & 1,751 & 14.57 & $<0.001$ \\
\hline & Social.Incubator & 2,751 & 0.03 & 0.971 \\
\hline & Landtype & 1,751 & 14.88 & $<0.001$ \\
\hline & $\rightarrow$ Social.Landtype & 1,751 & 2.02 & 0.159 \\
\hline & Population|Landtype(Mainland) & 2,751 & 4.38 & 0.013 \\
\hline & Population |Landtype(Island) & 3,751 & 29.71 & $<0.001$ \\
\hline & Social.Population|Landtype(Mainland) & 2,751 & 0.87 & 0.420 \\
\hline & Social.Population|Landtype(Island) & 3,751 & 1.43 & 0.234 \\
\hline \multirow[t]{10}{*}{ PC2 } & $\mu$ & 1,751 & 24.67 & $<0.001$ \\
\hline & PW & 1,751 & 59.69 & $<0.001$ \\
\hline & Social & 1,751 & 0.52 & 0.470 \\
\hline & Social.Incubator & 2,751 & 0.24 & 0.788 \\
\hline & Landtype & 1,751 & 64.75 & $<0.001$ \\
\hline & $\rightarrow$ Social.Landtype & 1,751 & 3.83 & 0.052 \\
\hline & Population|Landtype(Mainland) & 2,751 & 15.26 & $<0.001$ \\
\hline & Population |Landtype(Island) & 3,751 & 41.34 & $<0.001$ \\
\hline & Social.Population|Landtype(Mainland) & 2,751 & 0.14 & 0.870 \\
\hline & Social.Population |Landtype(Island) & 3,751 & 0.18 & 0.909 \\
\hline \multirow[t]{10}{*}{ PC3 } & $\mu$ & 1,751 & 6.97 & 0.009 \\
\hline & PW & 1,751 & 0.00 & 0.980 \\
\hline & Social & 1,751 & 27.63 & $<0.001$ \\
\hline & Social.Incubator & 2,751 & 0.71 & 0.495 \\
\hline & Landtype & 1,751 & 224.77 & $<0.001$ \\
\hline & $\rightarrow$ Social.Landtype & 1,751 & 0.20 & 0.648 \\
\hline & Population |Landtype(Mainland) & 2,751 & 0.39 & 0.679 \\
\hline & Population|Landtype(Island) & 3,751 & 23.54 & $<0.001$ \\
\hline & Social.Population |Landtype(Mainland) & 2,751 & 1.07 & 0.345 \\
\hline & Social.Population|Landtype(Island) & 3,751 & 0.95 & 0.419 \\
\hline
\end{tabular}




\begin{tabular}{|c|c|c|c|c|}
\hline \multirow[t]{10}{*}{ PC4 } & $\mu$ & 1,751 & 10.76 & 0.001 \\
\hline & PW & 1,751 & 2.44 & 0.121 \\
\hline & Social & 1,751 & 3.68 & 0.057 \\
\hline & Social.Incubator & 2,751 & 0.75 & 0.472 \\
\hline & Landtype & 1,751 & 64.68 & $<0.001$ \\
\hline & $\rightarrow$ Social.Landtype & 1,751 & 2.41 & 0.123 \\
\hline & Population|Landtype(Mainland) & 2,751 & 0.04 & 0.960 \\
\hline & Population |Landtype(Island) & 3,751 & 13.06 & $<0.001$ \\
\hline & Social.Population |Landtype(Mainland) & 2,751 & 3.07 & 0.048 \\
\hline & Social.Population|Landtype(Island) & 3,751 & 0.78 & 0.509 \\
\hline \multirow[t]{10}{*}{ PC5 } & $\mu$ & 1,751 & 0.29 & 0.588 \\
\hline & PW & 1,751 & 0.39 & 0.528 \\
\hline & Social & 1,751 & 0.79 & 0.375 \\
\hline & Social.Incubator & 2,751 & 0.38 & 0.681 \\
\hline & Landtype & 1,751 & 57.26 & $<0.001$ \\
\hline & $\rightarrow$ Social.Landtype & 1,751 & 0.05 & 0.817 \\
\hline & Population|Landtype(Mainland) & 2,751 & 0.28 & 0.757 \\
\hline & Population|Landtype(Island) & 3,751 & 1.58 & 0.194 \\
\hline & Social.Population |Landtype(Mainland) & 2,751 & 3.02 & 0.050 \\
\hline & Social.Population|Landtype(Island) & 3,751 & 0.45 & 0.717 \\
\hline \multirow[t]{10}{*}{ PC6 } & $\mu$ & 1,751 & 26.98 & $<0.001$ \\
\hline & PW & 1,751 & 4.17 & 0.043 \\
\hline & Social & 1,751 & 0.01 & 0.899 \\
\hline & Social.Incubator & 2,751 & 0.73 & 0.481 \\
\hline & Landtype & 1,751 & 5.04 & 0.026 \\
\hline & $\rightarrow$ Social.Landtype & 1,751 & 3.88 & 0.051 \\
\hline & Population|Landtype(Mainland) & 2,751 & 3.47 & 0.032 \\
\hline & Population |Landtype(Island) & 3,751 & 21.99 & $<0.001$ \\
\hline & Social.Population |Landtype(Mainland) & 2,751 & 0.98 & 0.376 \\
\hline & Social.Population|Landtype(Island) & 3,751 & 1.13 & 0.339 \\
\hline
\end{tabular}

Pascoal et al. Divergence and Social Flexibility page 63 of 65 
Table S8. Results of univariate linear mixed effect models (Model 2) showing estimated effect sizes and statistical inference. Note that effect size is given for the No Song treatment relative to the average in the Song treatment.

\begin{tabular}{|c|c|c|c|c|c|c|c|c|}
\hline \multirow[b]{2}{*}{ Trait } & \multirow[b]{2}{*}{ Effect } & \multicolumn{4}{|c|}{ Fixed effects } & \multicolumn{3}{|c|}{ Random effects } \\
\hline & & Coefficient (SE) & $F$ & $d f$ & $P$ & Proportion $V_{p}$ explained & $\chi_{1}^{2}$ & $P$ \\
\hline \multirow[t]{4}{*}{ PC1 } & $\mu$ & $0.120(0.141)$ & 0.01 & $1,5.9$ & 0.938 & & & \\
\hline & PW & $1.178(0.126)$ & 87.19 & 1,735 & $<.001$ & & & \\
\hline & Social (No Song) & $-0.229(0.060)$ & 14.42 & $1,759.2$ & $<.001$ & & & \\
\hline & Population & & & & & $0.126(0.077)$ & 83.2 & $<0.001$ \\
\hline \multirow[t]{4}{*}{ PC2 } & $\mu$ & $0.004(0.220)$ & 0 & $1,5.9$ & 0.957 & & & \\
\hline & PW & $1.018(0.134)$ & 57.6 & $1,763.7$ & $<.001$ & & & \\
\hline & Social (No Song) & $-0.047(0.064)$ & 0.54 & $1,759.1$ & 0.461 & & & \\
\hline & Population & & & & & $0.325(0.192)$ & 164.0 & $<0.001$ \\
\hline \multirow[t]{4}{*}{ PC3 } & $\mu$ & $0.135(0.241)$ & 0.01 & 1,6 & 0.932 & & & \\
\hline & PW & $0.011(0.122)$ & 0.01 & 1,765 & 0.913 & & & \\
\hline & Social (No Song) & $0.305(0.058)$ & 27.8 & 1,759 & $<.001$ & & & \\
\hline & Population & & & & & $0.395(0.232)$ & 222.0 & $<0.001$ \\
\hline \multirow[t]{4}{*}{ PC4 } & $\mu$ & $0.080(0.162)$ & 0.01 & $1,5.9$ & 0.941 & & & \\
\hline & PW & $-0.193(0.141)$ & 1.88 & $1,739.2$ & 0.173 & & & \\
\hline & Social (No Song) & $-0.129(0.068)$ & 3.62 & $1,759.1$ & 0.059 & & & \\
\hline & Population & & & & & $0.167(0.102)$ & 73.5 & $<0.001$ \\
\hline \multirow[t]{4}{*}{ PC5 } & $\mu$ & $-0.046(0.136)$ & 0.01 & $1,5.8$ & 0.916 & & & \\
\hline & PW & $0.042(0.143)$ & 0.09 & $1,691.7$ & 0.76 & & & \\
\hline & Social (No Song) & 0.060 (0.069) & 0.76 & $1,759.1$ & 0.385 & & & \\
\hline & Population & & & & & $0.113(0.071)$ & 38.6 & $<0.001$ \\
\hline \multirow[t]{4}{*}{ PC6 } & $\mu$ & $-0.005(0.130)$ & 0 & $1,5.9$ & 0.995 & & & \\
\hline & PW & $0.260(0.143)$ & 3.3 & $1,676.4$ & 0.072 & & & \\
\hline & Social (No Song) & $0.007(0.069)$ & 0.01 & $1,759.3$ & 0.913 & & & \\
\hline & Population & & & & & $0.102(0.064)$ & 54.1 & $<0.001$ \\
\hline
\end{tabular}


1

2

3

4

5

6

7

8

9

10

11

12

13

14

15

\section{LITERATURE CITED}

Bailey, N. W., and E. Macleod. 2014. Socially flexible female choice and premating isolation in field crickets (Teleogryllus spp.). Journal of Evolutionary Biology 27:170-180.

Goudet, J. 1995. FSTAT (version 1.2) A computer program to calculate F-statistics. Journal of Heredity 86:385-386.

Majlát, P., Z. Erdos, and J. Takacs. 1974. Calculation and application of retention indices in programmed-temperature gas-chromatography. Journal of Chromatography 91:89103.

Tabachnick, B. G., and L. S. Fidell. 1989. Using Multivariate Statistics ( $2^{\text {nd }}$ edition). Harper Collins Publishers, New York. 